\title{
DUST AND GAS IN THE MAGELLANIC CLOUDS FROM THE HERITAGE HERSCHEL KEY PROJECT. II. GAS-TO-DUST RATIO VARIATIONS ACROSS INTERSTELLAR MEDIUM PHASES
}

\author{
Julia Roman-Duval ${ }^{1}$, Karl D. Gordon ${ }^{1,2}$, Margaret Meixner ${ }^{1,3}$, Caroline Bot ${ }^{4}$, Alberto Bolatto ${ }^{5}$, Annie Hughes ${ }^{6}$, \\ Tony Wong ${ }^{7}$, Brian Babler ${ }^{8}$, Jean-Philippe Bernard ${ }^{9}$, Geoffrey C. Clayton ${ }^{10}$, Yasuo Fukui $^{11}$, Maud Galametz ${ }^{12}$, \\ Frederic Galliano $^{13}$, Simon Glover ${ }^{14}$, Sacha Hony ${ }^{6}$, Frank Israel ${ }^{15}$, Katherine Jameson $^{5}$, Vianney Lebouteiller ${ }^{13}$, \\ Min-Young LeE ${ }^{13}$, Aigen Li ${ }^{16}$, Suzanne Madden ${ }^{13}$, Karl Misselt $^{17}$, Edward Montiel ${ }^{10}$, Koryo Okumura $^{13}$, \\ Toshikazu Onishi ${ }^{18}$, Pasquale Panuzzo ${ }^{19}$, William Reach ${ }^{20}$, Aurelie Remy-Ruyer $^{13}$, Thomas Robitaille ${ }^{6}$, \\ Monica Rubio ${ }^{21}$, Marc Sauvage ${ }^{13}$, Jonathan Seale ${ }^{3}$, Marta Sewilo $^{3}$, \\ Lister Staveley-Smith ${ }^{22}$, AND Svitlana Zhukovska ${ }^{6,14}$ \\ ${ }^{1}$ Space Telescope Science Institute, 3700 San Martin Drive, Baltimore, MD 21218, USA; duval@ stsci.edu \\ ${ }^{2}$ Sterrenkundig Observatorium, Universiteit Gent, Gent, Belgium \\ ${ }^{3}$ Department of Physics and Astronomy, The Johns Hopkins University, 366 Bloomberg Center, 3400 N. Charles Street, Baltimore, MD 21218, USA \\ ${ }^{4}$ Observatoire astronomique de Strasbourg, Université de Strasbourg, CNRS, UMR 7550, 11 rue de l'université, F-67000 Strasbourg, France \\ ${ }^{5}$ Department of Astronomy, Lab for Millimeter-wave Astronomy, University of Maryland, College Park, MD 20742-2421, USA \\ ${ }^{6}$ Max-Planck-Institut für Astronomie, Königstuhl 17, D-69117 Heidelberg, Germany \\ ${ }^{7}$ University of Illinois at Urbana-Champaign, 1002 W. Green St., Urbana, IL 61801, USA \\ ${ }^{8}$ Department of Astronomy, University of Wisconsin, 475 North Charter St., Madison, WI 53706, USA \\ ${ }^{9}$ CNRS, IRAP, 9 Av. colonel Roche, BP 44346, F-31028 Toulouse Cedex 4, France \\ ${ }^{10}$ Louisiana State University, Department of Physics \& Astronomy, 233-A Nicholson Hall, Tower Dr., Baton Rouge, LA 70803-4001, USA \\ ${ }_{11}$ Department of Physics, Nagoya University, Chikusa-ku, Nagoya 464-8602, Japan \\ ${ }^{12}$ European Southern Observatory, Karl-Schwarzschild-Str 2, D-85748 Garching, Germany \\ ${ }^{13}$ CEA, Laboratoire AIM, Irfu/SAp, Orme des Merisiers, F-91191 Gif-sur-Yvette, France \\ ${ }^{14}$ Zentrum für Astronomie, Institut für Theoretische Astrophysik, Universität Heidelberg, Albert-Ueberle Strasse 2, D-69120 Heidelberg, Germany \\ ${ }^{15}$ Sterrewacht Leiden, Leiden University, P.O. Box 9513, NL-2300 RA Leiden, The Netherlands \\ ${ }^{16} 314$ Physics Building, Department of Physics and Astronomy, University of Missouri, Columbia, MO 65211, USA \\ ${ }^{17}$ Steward Observatory, University of Arizona, 933 North Cherry Ave., Tucson, AZ 85721, USA \\ ${ }^{18}$ Osaka Prefecture University, Department of Physical Science, 1-1 Gakuen-cho, Nakaku, Sakai, Osaka 599-853, Japan \\ ${ }^{19}$ CNRS, Observatoire de Paris-Lab. GEPI, Bat. 11, 5, place Jules Janssen, F-92195 Meudon CEDEX, France \\ ${ }^{20}$ Stratospheric Observatory for Infrared Astronomy, Universities Space Research Association, Mail Stop 232-11, Moffett Field, CA 94035, USA \\ ${ }^{21}$ Departamento de Astronomía, Universidad de Chile, Casilla 36-D, Santiago, Chile \\ 22 The University of Western Australia, 35 Stirling Highway, Crawley, WA 6009, Australia \\ Received 2014 January 24; accepted 2014 November 3; published 2014 December 3
}

\begin{abstract}
The spatial variations of the gas-to-dust ratio (GDR) provide constraints on the chemical evolution and lifecycle of dust in galaxies. We examine the relation between dust and gas at 10-50 pc resolution in the Large and Small Magellanic Clouds (LMC and SMC) based on Herschel far-infrared (FIR), H I $21 \mathrm{~cm}, \mathrm{CO}$, and H $\alpha$ observations. In the diffuse atomic interstellar medium (ISM), we derive the GDR as the slope of the dust-gas relation and find GDRs of $380_{-130}^{+250} \pm 3$ in the LMC, and $1200_{-420}^{+1600} \pm 120$ in the SMC, not including helium. The atomicto-molecular transition is located at dust surface densities of $0.05 M_{\odot} \mathrm{pc}^{-2}$ in the LMC and $0.03 M_{\odot} \mathrm{pc}^{-2}$ in the SMC, corresponding to $A_{V} \sim 0.4$ and 0.2 , respectively. We investigate the range of CO-to- $\mathrm{H}_{2}$ conversion factor to best account for all the molecular gas in the beam of the observations, and find upper limits on $X_{\mathrm{CO}}$ to be $6 \times 10^{20} \mathrm{~cm}^{-2} \mathrm{~K}^{-1} \mathrm{~km}^{-1} \mathrm{~s}$ in the $\mathrm{LMC}\left(Z=0.5 Z_{\odot}\right)$ at $15 \mathrm{pc}$ resolution, and $4 \times 10^{21} \mathrm{~cm}^{-2} \mathrm{~K}^{-1} \mathrm{~km}^{-1} \mathrm{~s}$ in the SMC $\left(Z=0.2 Z_{\odot}\right)$ at $45 \mathrm{pc}$ resolution. In the LMC, the slope of the dust-gas relation in the dense ISM is lower than in the diffuse ISM by a factor $\sim 2$, even after accounting for the effects of CO-dark $\mathrm{H}_{2}$ in the translucent envelopes of molecular clouds. Coagulation of dust grains and the subsequent dust emissivity increase in molecular clouds, and/or accretion of gas-phase metals onto dust grains, and the subsequent dust abundance (dust-to-gas ratio) increase in molecular clouds could explain the observations. In the SMC, variations in the dust-gas slope caused by coagulation or accretion are degenerate with the effects of $\mathrm{CO}$-dark $\mathrm{H}_{2}$. Within the expected 5-20 times Galactic $X_{\mathrm{CO}}$ range, the dust-gas slope can be either constant or decrease by a factor of several across ISM phases. Further modeling and observations are required to break the degeneracy between dust grain coagulation, accretion, and $\mathrm{CO}$-dark $\mathrm{H}_{2}$. Our analysis demonstrates that obtaining robust ISM masses remains a non-trivial endeavor even in the local Universe using state-of-the-art maps of thermal dust emission.
\end{abstract}

Key words: dust, extinction - ISM: clouds - ISM: molecules - ISM: structure

Online-only material: color figures

\section{INTRODUCTION}

The abundance and composition of dust in galaxies reflect the history of dust formation and dust destruction processes. Measurements of the gas-to-dust ratio (GDR) therefore provide important constraints on the chemical evolution of galaxies and the mechanisms responsible for their evolution (Dwek 1998). Dust formation results from condensation of metals in the atmospheres of evolved stars (Bladh \& Höfner 2012) and in the remnants of supernovae (Matsuura et al. 2011), where the 
high-density and low-temperature conditions are favorable to the condensation of refractory metals. In addition, dust could likely grow in the dense interstellar medium (ISM; Draine 2009; Zhukovska et al. 2008). Dust is predominantly destroyed via sputtering in shocks propagating through the ISM at speeds $\geqslant 100 \mathrm{~km} \mathrm{~s}^{-1}$ (e.g., Draine \& Salpeter 1979; Jones et al. 1994, 1996).

Dust grains play a central role in the radiative transfer, chemistry, and thermodynamics of the ISM, and therefore also in the star formation process. For example, molecular hydrogen $\left(\mathrm{H}_{2}\right)$, the fuel for star formation, forms on dust grains (Gould \& Salpeter 1963; Hill \& Hollenbach 1976). In addition, dust shields the molecular ISM from photo-dissociating UV radiation (Hollenbach \& Tielens 1999), allowing the gas to cool, fragment, and star formation to proceed (Krumholz et al. 2009b).

To understand the structure of the ISM and the lifecycle of dust, it is therefore essential to understand the variations of the dust abundance and composition as a function of environment and metallicity. Here, we carry out a study of the spatial variations of the ISM GDR in the Large and Small Magellanic Clouds (LMC and SMC). The Magellanic Clouds are ideal environments to constrain the variations of the GDR between ISM phases. They are relatively close, with the LMC at $50 \mathrm{kpc}$ (Schaefer 2008) and the SMC at $62 \mathrm{kpc}$ (Hilditch et al. 2005). The LMC has a thin-disk morphology, with the SMC having a thicker disk comparable to its projected extent on the sky (Subramanian \& Subramaniam 2009, 2010, 2012). The Magellanic Clouds have favorable viewing angle, with inclinations of $\sim 35^{\circ}$ for the LMC (van der Marel \& Cioni 2001) and $\sim 3^{\circ}$ for the SMC (Subramanian \& Subramaniam 2012). They span a range of environments (star forming and quiescent), and metallicities, from $Z=0.5 Z_{\odot}$ in the LMC down to $Z=$ $0.2 Z_{\odot}$ in the SMC (Russell \& Dopita 1992).

Constraining the variations of the GDR between ISM phases requires an accurate census of the dust and gas surface densities at spatial scales that resolve the cold phase, typically a few tens of parsecs (Roman-Duval et al. 2010). This is now possible with the combination of Herschel observations, which offer $8^{\prime \prime}-40^{\prime \prime}$ resolution (2-10 pc at the distance of the LMC), and groundbased observations of emission-based ISM tracers (CO $J=$ $1-0, \mathrm{H}_{\text {I }} 21 \mathrm{~cm}$, and $\mathrm{H} \alpha$ ) with $\leqslant 45$ pc resolution. In this paper, we use Herschel observations taken as part of the HERITAGE project (HERschel Inventory of The Agents of GalaxyEvolution, PI: Meixner; Meixner et al. 2013), which sampled the spectral energy distribution (SED) from ISM (10-100 K) dust in five bands $(100,160,250,350,500 \mu \mathrm{m})$. Compared to previous measurements of the dust content of the Magellanic Clouds from IRAS (Schwering \& Israel 1989; Schwering 1989) and Spitzer (Meixner et al. 2006; Bolatto et al. 2007; Gordon et al. 2011) observations, the Herschel observations represent a major step forward in terms of wavelength coverage.

The GDR definition is volumic: $\mathrm{GDR}=\rho_{\mathrm{gas}} / \rho_{\text {dust }}$, where $\rho_{\text {gas }}$ and $\rho_{\text {dust }}$ are the mass densities of gas and dust. Thus, GDR = $\left(d \Sigma_{\text {gas }} / d l\right) /\left(d \Sigma_{\text {dust }} / d l\right)=d \Sigma_{\text {gas }} / d \Sigma_{\text {dust }}$. Therefore, the GDR corresponds to the derivative (or slope) of the relation between dust and gas surface densities. This is the approach we take in the following to derive GDR values in the different ISM phases.

The paper is organized as follows. Section 2 discusses the observations of the dust and gas content of the LMC and SMC. Section 3 describes the methodology. Sections 3-5 describe the relation between dust and atomic, molecular, and total gas, respectively, from which we derive the diffuse and dense GDRs and the location of the atomic-molecular gas transition. In
Section 6, we compare our results to UV measurements of the GDR and to depletions studies. We also discuss the accuracy and interpretation of GDR maps obtained by taking the ratio of gas and dust surface densities. Finally, Section 7 summarizes the conclusions of the paper.

\section{OBSERVATIONS}

\subsection{Dust}

The dust surface density is estimated in a companion paper by Gordon et al. (2014, hereafter Paper I) using Herschel PACS and SPIRE observations of the Magellanic Clouds taken as part of the HERITAGE key project. The Herschel observations, presented in Meixner et al. (2013), include five bands at 100, 160, 250, 350, and $500 \mu \mathrm{m}$, and cover the Magellanic Clouds and the SMC Tail. Prior to the dust surface density derivation via SED fitting, Gordon et al. (2014) convolved the FIR maps from their native resolution of $8^{\prime \prime}, 12^{\prime \prime}, 18^{\prime \prime}, 25^{\prime \prime}$ for PACS 100, 160, SPIRE 250, 350 to the limiting resolution of the SPIRE 500 band. The data processing, uncertainties, and flux calibration errors are described in detail in Meixner et al. (2013).

Paper I takes a probabilistic approach to fitting the background-subtracted FIR Herschel photometry of each pixel to three different dust emission models: (1) a single-temperature blackbody modified by a single power-law emissivity $\beta$ (SMBB), (2) a single-temperature blackbody modified by a broken power-law emissivity (BEMBB), and (3) a two-temperature blackbody modified by a power-law emissivity (TTMBB). Paper I demonstrates that the SMBB and TTMBB produce worse fits, and that the global GDR $\left(\mathrm{M}_{\mathrm{gas}} / \mathrm{M}_{\text {dust }}\right)$ derived from the TTMBB model violates elemental abundance constraints in the LMC and SMC. Thus, we adopt the BEMBB model as our fiducial model in this study. In Appendix C, we investigate the robustness of the observed GDR trends against the choice of dust model, and we confirm that the TTMBB model violates elemental abundances. We show that the GDR values derived from the BEMBB and SMBB models are equivalent within the uncertainties.

The parameters of the BEMBB model are the modified blackbody temperature, $T_{\text {dust }}$, surface density $\Sigma_{\text {dust }}$, and the spectral indices of the broken emissivity law, $\beta_{1}$ for $\lambda \leqslant \lambda_{b}$ and $\beta_{2}$ for $\lambda>\lambda_{b}$, where $\lambda_{b}$ is the break wavelength at which the spectral emissivity index changes, and $\lambda_{b}$ is also a free parameter. The BEMBB assumes an opacity at $160 \mu \mathrm{m}$ $\kappa_{160}=11.6 \mathrm{~cm}^{2} \mathrm{~g}^{-1}$, which is calibrated using a diffuse solar neighborhood FIR SED to obtain a GDR of 150 (without the helium contribution). This GDR value is obtained from depletion measurements (Jenkins 2009) corresponding to the column density of hydrogen measured toward the same line-ofsight as the FIR SED (Gordon et al. 2014). Assuming a different value of $\kappa_{160}$ would systematically scale the dust surface density and GDR values accordingly. The systematic uncertainty on $\kappa_{160}$ is discussed in Appendix A.

Paper I derives two dust maps from the BEMBB model. In the first case, $\beta_{1}$ is left unconstrained. In the second case, $\beta_{1}$ is restricted to be between 0.8 and 2.5 . We choose to apply the uniform prior on $\beta_{1}$ between 0.8 and 2.5. This assumption is justified by laboratory measurements of $\beta$ (Coupeaud et al. 2011), and allows us to reduce the noise and scatter in the dust-gas relation.

The probabilistic framework outputs posterior probabilities. To generate a map of a parameter, one then must choose a representative value from its probability density function (PDF), 


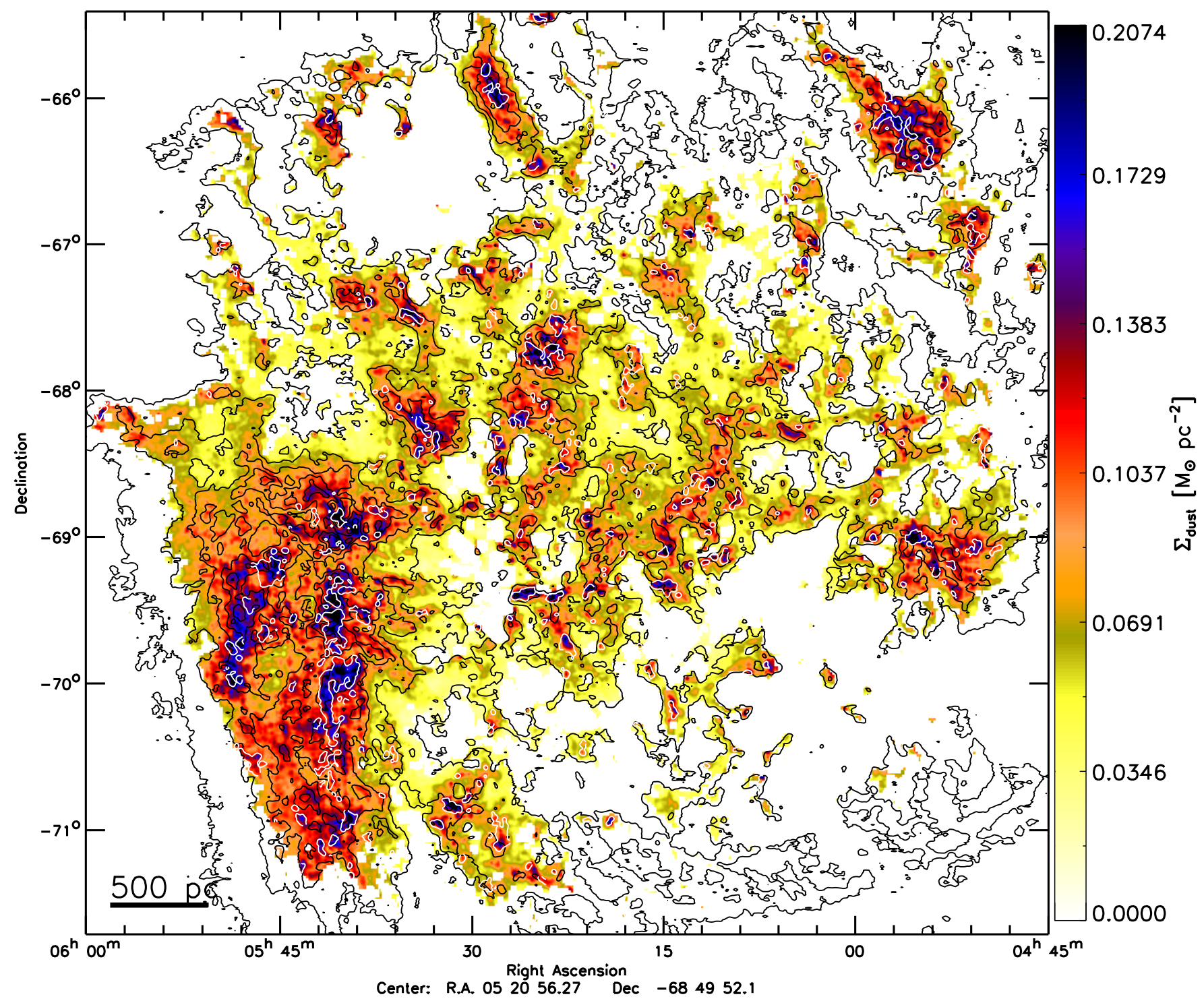

Figure 1. Map of the dust surface density in the LMC from Paper I at $1^{\prime}(15 \mathrm{pc})$ resolution. The black contours show the H I surface density (Kim et al. 2003), with levels $10-60 M_{\odot} \mathrm{pc}^{-2}$ in steps of $10 M_{\odot} \mathrm{pc}^{-2}$. The white contours show the CO integrated intensity (Wong et al. 2011), with level $1.2 \mathrm{~K} \mathrm{~km} \mathrm{~s}^{-1}$.

(A color version of this figure is available in the online journal.)

such as the expectation value, the median, maximum likelihood, or a random realization. Paper I performed a sensitivity analysis to establish which of these measurements minimizes the bias and scatter, and found that the expectation value provides the least biased and the most robust results. For the BEMBB model, they found that the expectation value of the dust surface density PDF recovers the dust surface density to within 5\%. Thus, in the rest of this analysis, we use the surface density maps derived by taking the expectation value of the marginalized posterior distributions for each of the parameters.

The dust surface density maps are shown in Figures 1 and 2. Paper I derived dust surface densities for pixels with FIR emission detected above $3 \sigma$ in all five bands. The total dust masses in the dust surface density maps are $(6.7 \pm 1.7)$ $\times 10^{5} M_{\odot}$ and $(6.7 \pm 1.7) \times 10^{4} M_{\odot}$ for the LMC and SMC, respectively. By combining this result with that from fitting an average (stacked) SED of the pixels with FIR emission less than $3 \sigma$ in one or more bands, Paper I derived total dust masses of $(7.3 \pm 1.7) \times 10^{5} M_{\odot}$ and $(8.3 \pm 2.1) \times 10^{4} M_{\odot}$ for the LMC and SMC, respectively. In this work, we apply a further sensitivity cut to the dust surface density maps, and only include pixels where the signal-to-noise ratio $(\mathrm{S} / \mathrm{N})$ on the dust surface density determination is greater than 2 . This retains $80 \%$ of the pixels with dust surface density determinations in the LMC, and $100 \%$ of the pixels with dust surface density determinations in the SMC.

\subsection{Atomic Gas}

The atomic gas surface density is derived from $21 \mathrm{~cm}$ line emission maps with $1^{\prime}$ resolution combining observations carried out at the Australian Telescope Compact Array and at the Parkes $64 \mathrm{~m}$ radio Telescope. The combination of the singledish and interferometric observations are described in Kim et al. (2003; LMC) and Stanimirovic et al. (1999; SMC). The H I column density maps are converted from the $21 \mathrm{~cm}$ emission under the assumption of an optically thin line (e.g., Bernard et al. 2008). This assumption may not be correct in regions of high $\mathrm{H} \mathrm{I}$ column density, where we may systematically underestimate the H I content (Dickey et al. 2000; Fukui et al. 2014). Accordingly, the SMC H I map is corrected for the effects of optical thickness 


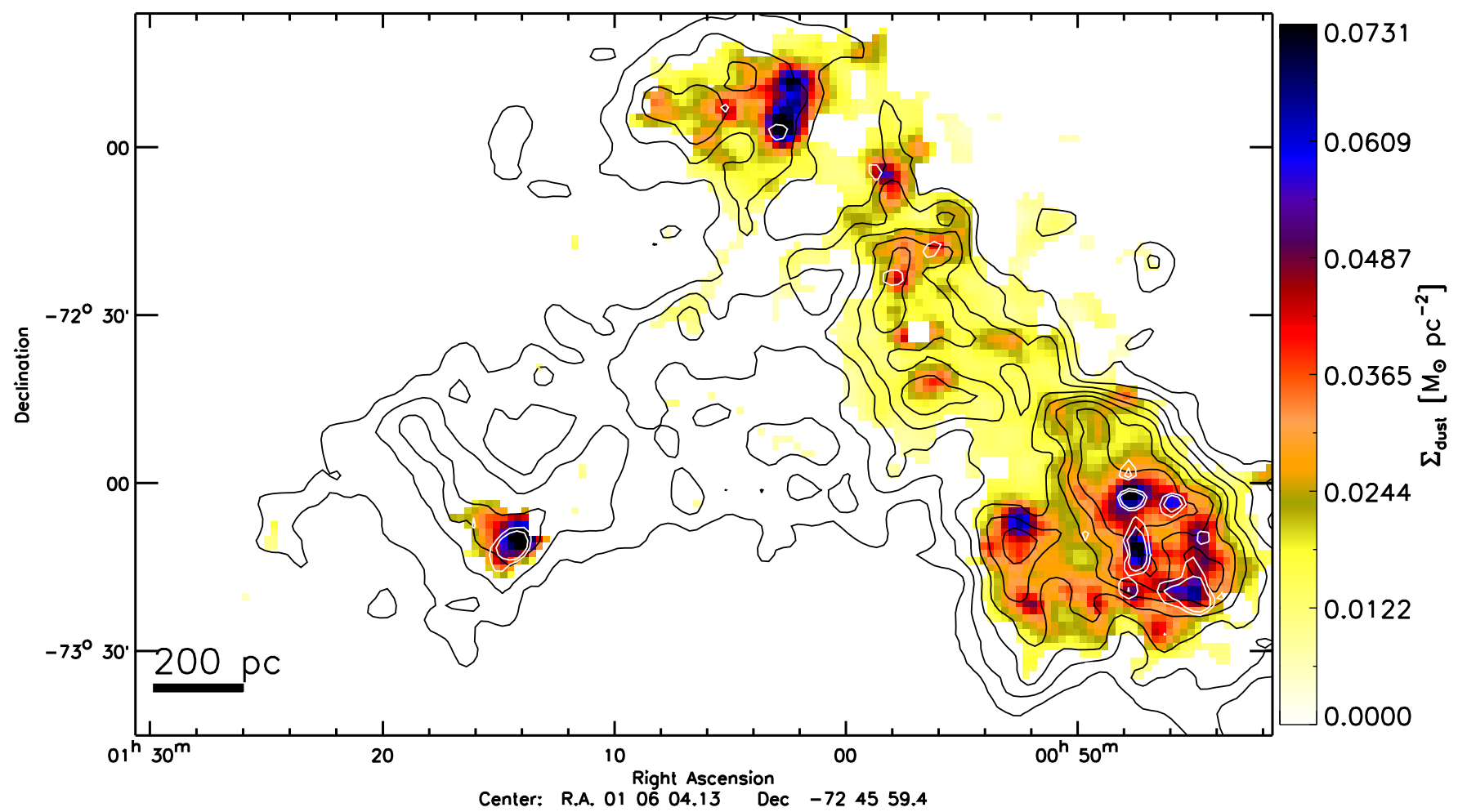

Figure 2. Map of the dust surface density in the SMC from Paper I at 2'.6 (45 pc) resolution. The black contours show the H I surface density (Stanimirovic et al. 1999), with levels $40-100 M_{\odot} \mathrm{pc}^{-2}$ in steps of $10 M_{\odot} \mathrm{pc}^{-2}$. The white contours show the CO integrated intensity (Mizuno et al. 2001a), with levels 0.5 and $0.8 \mathrm{~K} \mathrm{~km} \mathrm{~s}{ }^{-1}$. Note that the color scale is different from Figure 1 due to the lower dust abundance in the SMC.

(A color version of this figure is available in the online journal.)

and self-absorption as in Stanimirovic et al. (1999). For the LMC, such a correction is not known and cannot be applied.

The H I column density, $N(\mathrm{HI})$ in units of $\mathrm{cm}^{-2}$, is then converted to a surface density, $\Sigma(\mathrm{HI})$, via $\Sigma(\mathrm{HI})=0.8 \times 10^{-20}$ $N(\mathrm{HI})$, where $\Sigma(\mathrm{HI})$ is in $M_{\odot} \mathrm{pc}^{-2}$. The sensitivity of the $\mathrm{H}_{\mathrm{I}}$ maps is $\sim 1 M_{\odot} \mathrm{pc}^{-2}$. The $\mathrm{H}_{\mathrm{I}}$ surface density maps are shown as contours in Figures 1 and 2.

The total $\mathrm{H}$ I masses over our studied area are $2.4 \times 10^{8} M_{\odot}$ in the $\mathrm{LMC}$, and $1.1 \times 10^{8} M_{\odot}$ in the SMC which are $60 \%$ (LMC) and $35 \%$ (SMC) of the H I masses over the entire extent of the HERITAGE images. The masses reported here do not include the contribution of helium to the mean molecular weight (1.36).

\subsection{Molecular Gas}

\subsubsection{CO Observations}

The molecular gas component (or at least its densest contribution) is traced by its ${ }^{12} \mathrm{CO} J=1-0$ emission. We use two different CO surveys for the LMC and SMC. In the LMC, we rely on the MAGellanic Mopra Assessment (MAGMA) survey (Wong et al. 2011; http://mmwave.astro.illinois.edu/magma/) to trace molecular gas. The MAGMA project surveyed the ${ }^{12} \mathrm{CO}$ 1-0 line in the LMC with the $22 \mathrm{~m}$ MOPRA telescope of the Australia Telescope National Facility, with resolution of $45^{\prime \prime}$. The MAGMA survey was a targeted follow-up survey that obtained ${ }^{12} \mathrm{CO} 1-0$ maps of molecular regions with significant CO detections in a previous NANTEN CO 1-0 survey (Mizuno et al. 2001b), at 2'.6 resolution. Thus, the MAGMA survey does not blindly survey the whole LMC (see Hughes et al. 2010; Wong et al. 2011 for details on the observing strategy). As a result, the MAGMA survey is not complete, but accounts for most $(80 \%)$ of the CO luminosity in the LMC (Wong et al. 2011). For this analysis we use a new version of the MAGMA data (DR3) which includes additional regions mapped in 2012 and 2013, expanding the survey area by $\sim 20 \%$ compared to the Wong et al. (2011) data set. An integrated CO intensity image was obtained by applying a three-dimensional signal mask to the cube and summing pixels within the mask. The mask was derived by first smoothing the cube both spatially, to reach a resolution of $67 . .5$, and spectrally, by a convolving with a Gaussian with an FWHM of three channels $\left(1.5 \mathrm{~km} \mathrm{~s}^{-1}\right)$. Then the mask was generated by expanding from regions of high significance $(>5 \sigma)$ in the smoothed cube out to the $3 \sigma$ contour; both initial and expanded masks were required to be at least two channels wide at every spatial pixel. The uncertainty in the $\mathrm{CO}$ intensity was obtained from the position-dependent channel noise by considering the number of channels summed at each position in the mask, or by assuming a fiducial line width of $5 \mathrm{~km} \mathrm{~s}^{-1}$ outside the mask. The resulting $1 \sigma$ uncertainty ranges from 0.4 to $1 \mathrm{~K} \mathrm{~km} \mathrm{~s}^{-1}$.

In the SMC, the MAGMA ${ }^{12} \mathrm{CO}$ map is very incomplete, and so we make use of the NANTEN ${ }^{12} \mathrm{CO} 1-0$ map (Mizuno et al. 2001a) at 2'6 resolution instead. The NANTEN survey achieved a $1 \sigma$ sensitivity of $\sigma_{\mathrm{CO}}=0.07 \mathrm{~K} \mathrm{~km} \mathrm{~s}^{-1}$. Because of the coarser resolution in the SMC, we expect that $\mathrm{CO}$ clouds are not resolved.

For both surveys, the absolute flux calibration is accurate to $30 \%$. The ${ }^{12} \mathrm{CO}$ integrated intensity maps are shown in Figures 1 and 2 in the LMC and SMC. All of the CO emission lies in our area of study and there are no sensitivity cuts. The total $\mathrm{CO}$ luminosity of the LMC and SMC are $2.9 \times 10^{6} \mathrm{~K} \mathrm{~km} \mathrm{~s}^{-1} \mathrm{pc}^{2}$ and $1.8 \times 10^{5} \mathrm{~K} \mathrm{~km} \mathrm{~s}^{-1} \mathrm{pc}^{2}$, respectively. 


\subsection{2. $\mathrm{CO}$-dark $\mathrm{H}_{2}$ and $\mathrm{CO}$-to- $\mathrm{H}_{2}$ Conversion Factor (X Factor)}

In order to convert the observed $\mathrm{CO}$ integrated intensity into a column density of $\mathrm{H}_{2}$, one must assume a conversion factor, the so-called $X$ factor, defined as $X_{\mathrm{CO}}=\bar{N}\left(\mathrm{H}_{2}\right) / \bar{I}(\mathrm{CO})$. Here $\bar{N}\left(\mathrm{H}_{2}\right)$ and $\bar{I}(\mathrm{CO})$ are the average column density of $\mathrm{H}_{2}$ in $\mathrm{cm}^{-2}$ and the average ${ }^{12} \mathrm{CO}$ integrated intensity in $\mathrm{K} \mathrm{km} \mathrm{s}^{-1}$ in a given region. The general assumption is that the optically thick ${ }^{12} \mathrm{CO}$ 1-0 transition traces the molecular mass of a cloud because it arises from the surfaces of clumps within the telescope beam (the CO mist model discussed by Dickman et al. 1986). Hence, $I_{\mathrm{CO}}$ should be proportional to (1) the fraction of the beam filled with optically thick CO clumps, and (2) the mean $\mathrm{CO}$ brightness temperature of the various emitting components, which is set by the excitation of the molecule. The molecular gas surface density inferred from $\mathrm{CO}$ emission, $\Sigma\left(\mathrm{H}_{2}^{\mathrm{CO}}\right)$, is then:

$$
\Sigma\left(\mathrm{H}_{2}^{\mathrm{CO}}\right)=1.6 \times 10^{-20} X_{\mathrm{CO}} I_{\mathrm{CO}},
$$

where $\Sigma\left(\mathrm{H}_{2}^{\mathrm{CO}}\right)$ is in $M_{\odot} \mathrm{pc}^{-2}$ and $I_{\mathrm{CO}}$ is in $\mathrm{K} \mathrm{km} \mathrm{s}^{-1}$.

Because the $X$ factor is an empirical parameter, and because CO clumps do not usually fill the whole beam, a derived value of the $X$ factor depends on the resolution of the observations, and of the filling factor of the $\mathrm{CO}$ gas in the more extended volume of molecular gas. The latter is a function of metallicity, density, radiation field, and of the dynamical state of the $\mathrm{CO}$ clouds. As metallicity decreases and/or radiation field increases, the $\mathrm{CO}$ extended emission disappears, and the radius of $\mathrm{CO}$ clumps decreases due to the reduced level of dust shielding. In contrast, the radius of the $\mathrm{H}_{2}$ volume remains roughly the same, since $\mathrm{H}_{2}$ self-shields (Tielens \& Hollenbach 1985; Bolatto et al. 1999, 2008; Wolfire et al. 2010). Theoretical models (Wolfire et al. 2010; Glover \& Mac Low 2011) suggest that $\mathrm{H}_{2}$ can exist for extinctions as low as $A_{V}$ of 0.2 , while $\mathrm{CO}$ is photo-dissociated for extinctions lower than $A_{\mathrm{V}} \sim 1$ (Wolfire et al. 2010; Glover \& Mac Low 2011). The gas column density corresponding to this $\Delta A_{\mathrm{V}}^{\mathrm{H}_{2}, \mathrm{CO}}$ is inversely proportional to metallicity (Wolfire et al. 2010). As a result, the filling factor of $\mathrm{CO}$ gas within $\mathrm{H}_{2}$ clouds decreases as metallicity decreases, or radiation field increases. In addition, the abundance of $\mathrm{C}$ and $\mathrm{O}$ elements, and therefore also $\mathrm{CO}$, scales with metallicity.

There are two ways to conceptualize the $X$ factor, "resolved" and "unresolved." The "resolved" $X_{\mathrm{CO}}$ is measured or computed in the area where $\mathrm{CO}$ and $\mathrm{H}_{2}$ are co-spatial, in other words, in CO clumps. The "unresolved" $X_{\mathrm{CO}}$ applies within a region larger than $\mathrm{CO}$ clumps or clouds, which encompasses both unresolved $\mathrm{CO}$ clumps, and CO-dark molecular gas. Choosing either definition can lead to very different values of the $X$ factor. In the first case, the theoretical expectation is that the $X$ factor should be close to the Milky Way (MW) value for the following three reasons. The ${ }^{12} \mathrm{CO}$ line is optically thick. The ensemble properties of clouds as derived from their $\mathrm{CO}$ emission are observed to be roughly uniform across a sample of galaxy disks (Bolatto et al. 2008). Third, the $X$ factor measured in CO-bright regions is not expected to depend strongly on metallicity. On the other hand, "unresolved" $X_{\mathrm{CO}}$ values do depend strongly on metallicity, radiation field, and resolution through the filling factor of $\mathrm{CO}$ clumps with respect to $\mathrm{H}_{2}$ clouds. A beam larger than the size of CO clumps may contain a significant amount of CO-dark molecular gas at low metallicity, which will increase the $X$ factor compared to the resolved definition. Values of the $X_{\mathrm{CO}}$ reported in the literature based on $\mathrm{CO}$ transitions in the LMC range from twice to seven times the Galactic value (Israel 1997; Fukui et al. 2008; Hughes et al. 2010). In the SMC, reported values are 30-60 times the Galactic value (Israel 1997; Leroy et al. 2007, 2011).

Since CO clumps should be resolved in the LMC at $1^{\prime}$ $(15 \mathrm{pc})$ resolution, a reasonable assumption is that $X_{\mathrm{CO}}=2$ $\times 10^{20} \mathrm{~cm}^{-2} \mathrm{~K}^{-1} \mathrm{~km}^{-1} \mathrm{~s}$ (Bolatto et al. 2013). In the SMC, CO clumps are not resolved at 2'.6 (45 pc) resolution, particularly because $\mathrm{CO}$ clouds are expected to be smaller in the SMC compared to the MW due to the reduced level of shielding (Bolatto et al. 1999). Bolatto et al. (2013) give an estimate of the unresolved $X_{\mathrm{CO}}$ as a function of metallicity in their Equation (31). In the case where the total gas surface density of the disk is $\leqslant 100 M_{\odot} \mathrm{pc}^{-2}$, and applying some conversions, we get:

$$
\frac{X_{\mathrm{CO}}\left(Z^{\prime}\right)}{X_{\mathrm{CO}}(\mathrm{MW})}=0.67 \exp \left(\frac{0.4}{Z^{\prime} \Sigma_{\mathrm{GMC}, 100}}\right) \text {, }
$$

where $X_{\mathrm{CO}}\left(Z^{\prime}\right)$ and $X_{\mathrm{CO}}(\mathrm{MW})$ are the $X$ factors at metallicity $Z^{\prime}$ and in the $\mathrm{MW}$, and $\Sigma_{\mathrm{GMC}, 100}$ is the molecular cloud surface density in $100 M_{\odot} \mathrm{pc}^{-2}$. With $Z^{\prime}=0.2$ as in the SMC, and $\Sigma_{\mathrm{GMC}, 100}=1$ (Heyer et al. 2009), we find that $X_{\mathrm{CO}}(\mathrm{SMC}) /$ $X_{\mathrm{CO}}(\mathrm{MW})=5$. However, the exponential makes this estimate very uncertain and the values of $X_{\mathrm{CO}}(\mathrm{SMC}) / X_{\mathrm{CO}}(\mathrm{MW})$ could range between 2 and 40 because $\Sigma_{\mathrm{GMC}, 100}$ may range from 0.5 to 2 .

Nevertheless, we start with fiducial values $X_{\mathrm{CO}}^{\mathrm{fid}}=2 \times$ $10^{20} \mathrm{~cm}^{-2} \mathrm{~K}^{-1} \mathrm{~km}^{-1} \mathrm{~s}$ in the LMC and $X_{\mathrm{CO}}^{\mathrm{fid}}=1 \times$ $10^{21} \mathrm{~cm}^{-2} \mathrm{~K}^{-1} \mathrm{~km}^{-1} \mathrm{~s}$ in the SMC. With those values of $X_{\mathrm{CO}}$, the CO-bright $\mathrm{H}_{2}$ masses of the LMC and SMC are $9.2 \times$ $10^{6} M_{\odot}$ and $2.9 \times 10^{5} M_{\odot}$, respectively. We review later (Section 5.2) the influence of the choice of $X_{\mathrm{CO}}$ on the relation between dust and gas, and we constrain the plausible range of $X_{\mathrm{CO}}$ to best account for the $\mathrm{H}_{2}$.

In this study, our goal is to determine a value of $X_{\mathrm{CO}}$ that works on average for each galaxy. Therefore, we use a single $X_{\mathrm{CO}}$ value for each galaxy. We expect cloud-to-cloud variations in $X_{\mathrm{CO}}$ due to differences in the radiation field and dynamic environment would produce scatter in the relation between dust and gas surface densities. However, we do not attempt to constrain variations of $X_{\mathrm{CO}}$ with environment other than metallicity in this study.

\subsection{Ionized Gas}

The ionized gas component, which can have a significant contribution in massive star formation regions such as 30 Doradus (30 Dor), is traced by its $\mathrm{H} \alpha$ emission observed in the Southern H-Alpha Sky Survey Atlas (Gaustad et al. 2001), carried out at Cerro Tololo Inter-American Observatory in Chile. The resolution of the $\mathrm{H} \alpha$ image is 0.8. The $\mathrm{H} \alpha$ is converted to an $\mathrm{H}^{+}$column density following the method outlined by Paradis et al. (2011), who derived electron densities for different $\mathrm{H} \alpha$ brightness regimes (diffuse ionized gas, H II regions and very bright $\mathrm{H}$ II regions). The $\mathrm{H}^{+}$surface density is then $\Sigma\left(\mathrm{H}^{+}\right)=0.8 \times 10^{-20} N\left(\mathrm{H}^{+}\right)$. The uncertainty of the $\mathrm{H} \alpha$ intensity corresponds to a $1 \sigma$ uncertainty of the $\mathrm{H}^{+}$surface density of $0.38 M_{\odot} \mathrm{pc}^{-2}$ for both galaxies.

The total masses of ionized hydrogen in the pixels where a dust surface density can be derived are $7.2 \times 10^{6} M_{\odot}$ in the LMC and $8.2 \times 10^{5} M_{\odot}$ in the SMC.

\subsection{Convolution to Common Resolution}

All maps $\left(\Sigma(\mathrm{HI}), \Sigma\left(\mathrm{H}_{2}^{\mathrm{CO}}\right), \Sigma\left(\mathrm{H}^{+}\right)\right.$, and $\left.\Sigma_{\text {dust }}\right)$ were convolved to the limiting resolution of the $\mathrm{CO}$ (SMC, 2!6 or $45 \mathrm{pc}$ ) or $\mathrm{H} \mathrm{I}_{\mathrm{I}}$ 
Table 1

Parameters of the Fits to the Dust-Gas Relation (with the BEMBB Dust Model)

\begin{tabular}{|c|c|c|c|c|c|c|}
\hline \multirow[t]{2}{*}{ Galaxy } & \multirow[t]{2}{*}{ Dust Model } & \multicolumn{3}{|c|}{ Diffuse ISM Parameters } & \multicolumn{2}{|c|}{ Dense ISM Parameters } \\
\hline & & $\mathrm{GDR}^{\mathrm{dif}}$ & $\begin{array}{c}\Sigma_{I}^{\mathrm{a}} \\
\left(M_{\odot} \mathrm{pc}^{-2}\right)\end{array}$ & $\begin{array}{c}\sum_{d}^{\mathrm{dif} \mathrm{b}} \\
\left(M_{\odot} \mathrm{pc}^{-2}\right)\end{array}$ & $\delta_{\min }^{\text {dense } c, d}$ & $\delta_{\min }^{\text {dense e }}$ \\
\hline LMC & BEMBB & $380 \pm 3.1(-11 \%)$ & $9.8 \pm 0.06(-0.53 \%)$ & $0.05 \pm 0.001(1.4 \%)$ & $73 \pm 3.2(22 \%)$ & $190 \pm 16(-23 \%)$ \\
\hline SMC & BEMBB & $1200 \pm 120(-16 \%)$ & $39 \pm 2.0(6.7 \%)$ & $0.03 \pm 0.006(-7.1 \%)$ & $580 \pm 30(-9.1 \%)$ & $1200 \pm 40(-25 \%)$ \\
\hline
\end{tabular}

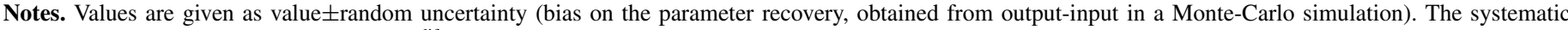
uncertainty on the dust-gas slopes and on $\Sigma_{d}^{\text {dif }}$ is $35 \%$ (see the Appendix A).

${ }^{\text {a }} \Sigma_{I}$ is the intercept of the dust-gas relation.

b $\Sigma_{d}^{\text {dif }}$ is the dust surface density corresponding to the $\mathrm{H} \mathrm{I}-\mathrm{H}_{2}$ transition.

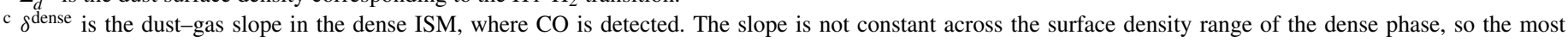
relevant, minimum value obtained at the highest surface densities is quoted.

${ }^{\mathrm{d}} X_{\mathrm{CO}}^{\text {fid }}=2 \times 10^{20} \mathrm{~cm}^{-2} \mathrm{~K}^{-1} \mathrm{~km}^{-1} \mathrm{~s}$ assumed in the LMC, and $X_{\mathrm{CO}}^{\mathrm{fid}}=1 \times 10^{21} \mathrm{~cm}^{-2} \mathrm{~K}^{-1} \mathrm{~km}^{-1} \mathrm{~s}$ in the SMC.

${ }^{\mathrm{e}} X_{\mathrm{CO}}^{\max }=6 \times 10^{20} \mathrm{~cm}^{-2} \mathrm{~K}^{-1} \mathrm{~km}^{-1} \mathrm{~s}$ assumed in the LMC, and $X_{\mathrm{CO}}^{\max }=4 \times 10^{21} \mathrm{~cm}^{-2} \mathrm{~K}^{-1} \mathrm{~km}^{-1} \mathrm{~s}$ in the SMC.

(LMC, $1^{\prime}$ or 15 pc) observations. We used a Gaussian kernel, of FWHM equal to the quadratic difference between the final and original resolutions, to perform the convolution. The maps were then resampled onto a common astrometric grid, of pixel size $30^{\prime \prime}$ in the LMC and $78^{\prime \prime}$ in the SMC (one-half of the PSF FWHM at limiting resolution).

We emphasize the importance of performing the FIR SED fitting to derive dust surface density maps at the best possible resolution, prior to convolving the dust surface density maps to the limiting resolution of the data set. In the LMC, Galliano et al. (2011) demonstrated that degrading the resolution of FIR maps from which dust masses are derived results in a significant bias in the resulting dust masses due to the combination of the dilution of cold regions into hotter regions at degraded resolution, and the non-linear (exponential, in fact) dependence of the IR flux on dust temperature. At $50 \mathrm{pc}$ resolution as in the SMC, the bias on the total dust mass amounts to $\sim-5 \%$ in our analysis. In Appendix D, we investigate the effects of convolving FIR maps before performing the SED fitting on the detailed pixel distribution, and show that the bias in individual pixels at low to intermediate surface densities can be as high as $100 \%$. Therefore, one should convolve the dust surface density maps, which are a linear tracer of the ISM, not the FIR flux or surface brightness maps. This is particularly important when using a single temperature dust model, as in this analysis.

\subsection{Total Gas}

The total gas surface density, not including helium, is the sum of the different gas components (atomic, molecular, ionized) described above: $\Sigma_{\text {gas }}=\Sigma\left(\mathrm{H}_{2}^{\mathrm{CO}}\right)+\Sigma\left(\mathrm{H}^{+}\right)+\Sigma(\mathrm{H} \mathrm{I})$, where we assume the fiducial $X$ factor values $X_{\mathrm{CO}}^{\mathrm{fid}}$. The total gas masses in the area where the FIR emission is $\geqslant 3 \sigma$ in all bands and a dust surface density can be derived are $2.4 \times 10^{8} M_{\odot}$ in the LMC, and $1.1 \times 10^{8} M_{\odot}$ in the SMC. Taking the ratio of the total gas and dust masses over the FIR-detected area yields GDRs of 350 and 1500 in the LMC and SMC, respectively. The total gas-total dust ratio over the entire extent of the HERITAGE survey yields GDRs of 550 in the LMC and 3900 in the LMC and SMC, respectively.

\section{RELATION BETWEEN DUST AND ATOMIC GAS}

\subsection{Method}

We first characterize the pixel-to-pixel relation between the dust surface density, $\Sigma_{\text {dust }}$, and the atomic + ionized hydrogen surface density, $\Sigma\left(\mathrm{H}_{\mathrm{I}}\right)+\Sigma\left(\mathrm{H}^{+}\right)$(Figure 3$)$. The correlation between dust and atomic + ionized gas surface densities appears linear up to dust surface densities $\Sigma_{d}^{\text {dif }} \simeq 0.05 M_{\odot} \mathrm{pc}^{-2}$ in the LMC and $\Sigma_{d}^{\text {dif }} \simeq 0.03 M_{\odot} \mathrm{pc}^{-2}$ in the SMC, above which the dust-H I gas relation flattens out, and saturates at $\mathrm{H}_{\mathrm{I}}$ surface densities of $40 M_{\odot} \mathrm{pc}^{-2}$ and $70 M_{\odot} \mathrm{pc}^{-2}$ in the LMC and SMC, respectively.

We determine in a quantitative way the location of the turnover in the dust-H I relation, $\Sigma_{d}^{\text {dif }}$, by fitting a broken linear function to the dust-H I pixel distribution. We only include pixels with dust surface density determination with $\mathrm{S} / \mathrm{N} \geqslant 2$. The broken linear function takes the form:

$$
\Sigma(\mathrm{HI})=\left\{\begin{array}{l}
\delta^{\text {dif }} \Sigma_{\text {dust }}+\Sigma_{I} \quad \text { if } \Sigma_{\text {dust }} \leqslant \Sigma_{\mathrm{d}}^{\text {dif }} \\
\delta^{\text {trans }}\left(\Sigma_{\text {dust }}-\Sigma_{d}^{\text {dif }}\right)+\Sigma_{I}+\delta^{\text {dif }} \Sigma_{d}^{\text {dif }} \quad \text { if } \Sigma_{\text {dust }} \geqslant \Sigma_{d}^{\text {dif }},
\end{array}\right.
$$

where $\delta^{\text {dif }}$ and $\delta^{\text {trans }}$ are the slopes of the dust-atomic gas relation below and above the threshold dust surface density $\Sigma_{d}^{\text {dif }} . \Sigma_{I}$ is the intercept of the correlation. We leave $\delta^{\mathrm{dif}}, \delta^{\text {trans }}, \Sigma_{I}$, and $\Sigma_{d}^{\mathrm{dif}}$ as free parameters of the fit, performed using a $\chi^{2}$ minimization to the functional form described in Equation (3). The parameters of this fit are reported in Table 1, and shown in Figure 3.

We have performed Monte-Carlo simulations to propagate the random errors on the dust and gas surface densities in the fitting, which produce both a bias and a random error on the dust-gas slope determination. The random error can be estimated as the standard deviation of the dust-gas slopes of all the realizations in the simulation. The bias is estimated as the difference between the mean of the outputs in the simulations and the input. For our fiducial dust model (BEMBB), the bias on $\delta^{\text {dif }}$ is $\sim 10 \%-15 \%$. The random uncertainty and biases on the parameter recovery are listed in Table 1. Table 1 also includes the systematic uncertainty on the dust surface density determination, which is derived in Appendix A. In Appendix B, we also explore the systematic uncertainty in the derived dust-gas slopes caused by the fitting method, and find that $\delta^{\text {dif }}$ is in the range 380-540 in the LMC, and 1200-2100 in the SMC for a wide variety of fitting techniques.

\section{2. $\mathrm{HI}-\mathrm{H}_{2}$ Boundary}

The dust surface density $\Sigma_{d}^{\text {dif }}$ at which the dust-atomic gas relation starts to flatten out coincides with the expected boundary between the diffuse atomic and diffuse molecular ISM (Krumholz et al. 2009a; Wolfire et al. 2010). We derive values 

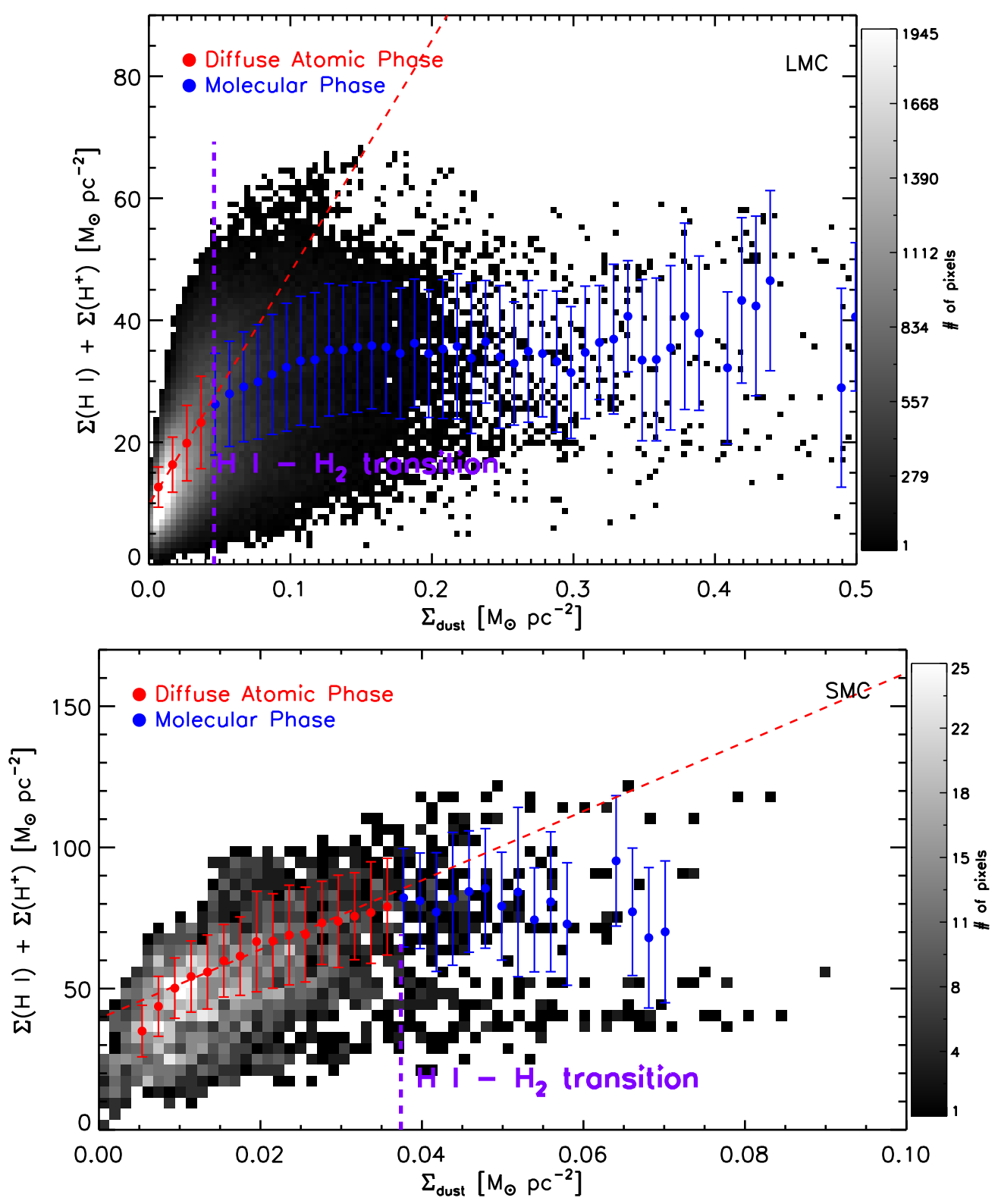

Figure 3. Pixel-to-pixel correlation between dust and atomic + ionized gas surface densities in the LMC (top) and SMC (bottom). The grayscale shows the density of points. The circles show the binned mean. The red and blue colors correspond to the atomic and molecular phases, respectively (see the text). The purple dashed line notes the transition point between atomic and molecular. The red dashed line corresponds to a linear fit to the dust-gas slope in the atomic phase, with a derived slope of $\delta^{\text {dif }}$ (reported in Table 1). See the text and Table 1 for details.

(A color version of this figure is available in the online journal.)

of $\Sigma_{d}^{\mathrm{dif}}=0.05 M_{\odot} \mathrm{pc}^{-2}$ in the LMC and $\Sigma_{d}^{\mathrm{dif}}=0.03 M_{\odot} \mathrm{pc}^{-2}$ in the SMC, which correspond to $A_{\mathrm{V}}=0.4$ and 0.2 , respectively, assuming a visual extinction efficiency $Q_{\mathrm{V}}=1.4$ (Chlewicki 1985). In comparison, Krumholz et al. (2009a), Wolfire et al. (2010), and Glover \& Mac Low (2011) predict that the $\mathrm{H} \mathrm{I}-\mathrm{H}_{2}$ boundary lies at a depth given by its visual extinction $A_{\mathrm{V}} \sim$ $0.2-0.3$, in good agreement with our derived values.

In addition, using FUV spectroscopy to measure $\mathrm{H}_{2}$ column densities toward a sample of LMC and SMC massive stars, Tumlinson et al. (2002) determined that the $\mathrm{H} \mathrm{I}-\mathrm{H}_{2}$ boundary is located at $E(B-V) \simeq 0.1$ in the $\mathrm{LMC}$ and $E(B-V) \simeq 0.06$ in the SMC. Assuming a ratio of total-to-selective extinction $R_{\mathrm{V}}=3.1$ in the LMC, and $R_{\mathrm{V}}=2.7$ in the SMC (Gordon et al. 2003), and an extinction efficiency $Q_{\mathrm{V}}=1.4$ (Chlewicki 1985), this corresponds to $\Sigma_{\text {dust }}=0.04 M_{\odot} \mathrm{pc}^{-2}$ and $0.02 M_{\odot} \mathrm{pc}^{-2}$, respectively, in excellent agreement with our measurements.
The $\mathrm{HI}-\mathrm{H}_{2}$ boundary appears to be located at increasingly lower $A_{\mathrm{V}}$ with decreasing metallicity. For comparison, Lee et al. (2012) found that the $\mathrm{HI}-\mathrm{H}_{2}$ boundary in the Perseus molecular cloud is located at $A_{\mathrm{V}}=0.5-0.8$. The dependency of the location of the $\mathrm{HI}-\mathrm{H}_{2}$ boundary is expected from theoretical models, such as Krumholz et al. (2009a). Thus, it is very likely that the flattening of the dust-atomic gas relation is caused by the gas turning molecular. Accordingly, we refer to pixels with dust surface densities below and above $\Sigma_{d}^{\text {dif }}$ as the diffuse and molecular phases, respectively, adopting the nomenclature from Snow \& McCall (2006).

\subsection{The Diffuse Atomic Gas-to-dust Ratio}

In the diffuse atomic phase, all the gas should be accounted for by the $\mathrm{H} \mathrm{I}$ and $\mathrm{H} \alpha$ observations, and we do not expect dust coagulation to occur. Therefore, $\delta^{\text {dif }}=\mathrm{GDR}^{\mathrm{dif}}$ is the diffuse atomic 
ISM GDR, with values $380_{-130}^{+250} \pm 3$ in the LMC ( \pm systematic uncertainty \pm random uncertainty), and $1200_{-420}^{+1600} \pm 120$ in the SMC. The quoted systematic uncertainty includes contributions from the uncertainty on the dust emissivity and the fitting method (see Appendices A and B).

We note that the value of $\delta^{\text {trans }}$ is irrelevant and does not have a physical meaning, since it is measured from the atomic gas in the molecular phase.

\subsection{Origin of the H I Pedestal}

Due to the relative nature of FIR observations, Paper I performed the same background subtraction procedure on the FIR and Hi maps, effectively setting the zero-point of the FIR and $\mathrm{H}_{\mathrm{I}} 21 \mathrm{~cm}$ emission at the edges of the maps, i.e., in the outskirts of the Magellanic Clouds. Therefore, the pedestal in the dust-gas relation could not be due to an offset between the FIR and Hi maps.

The non-zero intercept in the dust-H I gas relation, $\Sigma_{I}$, is likely due to a dust-poor diffuse gas component surrounding the FIR-bright regions of the LMC and SMC that is below the sensitivity of the FIR maps. The dust surface density maps only include pixels where the brightness is $\geqslant 3 \sigma$ in all five bands. Thus, the values of the diffuse GDR derived in Section 3.1 are in principle only applicable to pixels above this sensitivity cut. Calculating an intercept is equivalent to extrapolating a linear dust-gas relation below the sensitivity cut, and thus to assuming that the GDR of the FIR-faint regions is similar to the regions detected in the FIR.

However, we do know that both the $\mathrm{H}_{\mathrm{I}}$ and FIR maps reach zero emission levels in the outskirts of the Magellanic Clouds. Therefore, the dust-gas relation must go through the $\left(\Sigma_{\text {dust }}\right.$, $\left.\Sigma_{\text {gas }}\right)=(0,0)$ point, which requires a break in the dust-gas relation at the lowest dust surface densities observed with HERITAGE. The dust-gas slope, or GDR, below the minimum dust surface density measured in HERITAGE would then be steeper than the slope GDR ${ }^{\text {dif }}$ measured in the diffuse ISM where FIR emission is detected. This effect is evident in the SMC (Figure 3) where the lowest surface density pixels detected in HERITAGE already suggest a break in the slope of the dust-H I relation below $\Sigma_{\text {dust }}$ of $5 \times 10^{-3} M_{\odot} \mathrm{pc}^{-2}$. By taking the ratio $\Sigma_{\text {gas }}^{\min } / \Sigma_{\text {dust }}^{\min }$, where $\Sigma_{\text {gas }}^{\min }$ and $\Sigma_{\text {dust }}^{\min }$ are the minimum gas and dust surface densities measured in the $\mathrm{H}_{\mathrm{I}} 21 \mathrm{~cm}$ and FIR maps, we estimate that the GDR of the FIR-faint component would then be $\sim 3000-5000$ in the LMC and $~ 5000-10,000$ in the SMC.

Paper I performed a stacking analysis of the pixels of the FIR maps where the brightness is below the sensitivity cut and a dust surface density cannot be derived. They summed the FIR fluxes of those FIR-faint pixels, and fit the resulting SED to the BEMBB model. They found that the dust mass associated with this FIR-faint component is $(5.9 \pm 3.6) \times 10^{4} M_{\odot}$ in the LMC and $(1.6 \pm 1.3) \times 10^{4} M_{\odot}$ in the SMC. The corresponding H I masses are $1.6 \times 10^{8} M_{\odot}$ and $2.0 \times 10^{8} M_{\odot}$ in the LMC and SMC. The global $M_{\text {gas }} / M_{\text {dust }}$ ratios of this component are therefore $\sim 2800$ in the LMC and $\sim 12,000$ in the SMC, which is in reasonable agreement with the values derived by forcing the dust-gas relation to go through the $(0,0)$ point.

The presence of a dust-poor, FIR-faint component in the LMC and SMC is not implausible. It is known that the H I disk of star forming galaxies extends much beyond the FIR emitting central regions. For instance, Draine et al. (2007) find that the H I mass of a galaxy associated with FIR emission roughly corresponds to $30 \%$ of its total H I mass. This effect is exacerbated in the LMC and SMC by the tidal interaction between the clouds and the MW, which pulled out gas from the SMC 1.5-2.5 Gyr ago, when its metallicity was lower (Fox et al. 2013). The metallicity of the Magellanic Stream (MS) associated with the SMC is measured to be 0.1 solar (Fox et al. 2013). Assuming the GDR scales with metallicity as derived in Rémy-Ruyer et al. (2014, Table 1), the GDR of a 0.1 solar metallicity component would then be $\sim 8500$. If the FIR-faint SMC component had the same tidal origin and metallicity as the MS, its GDR value we derive would thus be in reasonable agreement with the measured metallicity of the MS. The metallicity of the part of the MS associated with the LMC has been measured toward one sight-line to be 0.1-0.5 solar, depending on the element (Richter et al. 2013), for which the GDR should be in the range 400-8500 from the GDR-metallicity relation from Rémy-Ruyer et al. (2014). In this case, the complex chemical enrichment history of the LMC-associated MS makes it difficult to predict the GDR. The GDR of the FIR-faint LMC component is compatible with the metallicity of the MS associated with the LMC, but remains poorly constrained due to the very large uncertainties.

We note that we cannot measure the $\mathrm{HI}_{\mathrm{I}}$ and dust masses of this component with great accuracy at the moment, because the absolute level of the FIR maps is not known, which requires us to force the zero point of the FIR and H I maps at the edges of the maps. Thus, the quoted H I and dust masses of the dust poor component represent lower limits. In the future, we will tie the FIR observations to previous COBE and Planck allsky observations in order to measure the absolute level of FIR emission in the outskirts of the Magellanic Clouds. We will then be able to measure the dust and gas masses of the FIR-weak component more accurately.

\section{RELATION BETWEEN DUST AND CO EMISSION}

Next, we examine the relation between dust and $\mathrm{CO}$ emission. The pixel-to-pixel correlation between $\Sigma_{\text {dust }}$ and $I_{\mathrm{CO}}$ is shown in Figure 4. The dust-CO relation appears linear at the highest surface densities, but is steeper at the lowest surface densities. We fit a linear function to pixels with $\mathrm{CO}$ detections and a dust surface density determined with $\mathrm{S} / \mathrm{N} \geqslant 2$ to determine the slope $\delta^{\mathrm{CO}}$ of the dust-CO relation. We take as the $\mathrm{CO}$ detection limit the $3 \sigma_{\mathrm{CO}}$ noise level in the SMC, and the detection limit in the $\mathrm{CO}$ integrated intensity determined by the masking algorithm in the LMC. We find $\delta^{\mathrm{CO}} \simeq 25 \mathrm{~K} \mathrm{~km} \mathrm{~s}^{-1} M_{\odot}{ }^{-1} \mathrm{pc}^{2}$ in the LMC, and $\delta^{\mathrm{CO}} \simeq 13 \mathrm{~K} \mathrm{~km} \mathrm{~s}^{-1} M_{\odot}{ }^{-1} \mathrm{pc}^{2}$ in the SMC. To gauge the systematic uncertainty on the dense dust-CO slope associated with the fitting method, we also performed a bisector fit to the dust-CO distribution, and found $\delta^{\mathrm{CO}}$ values that are within $10 \%$ of the values obtained with a linear fit. From the dust-CO slopes, we conclude that the $\mathrm{CO}$ emission per unit dust mass is twice as high in the LMC compared to the SMC.

On average, significant $\mathrm{CO}$ emission occurs for dust surface densities $\geqslant 0.05 M_{\odot} \mathrm{pc}^{-2}$ in the LMC, and $\geqslant 0.03 M_{\odot} \mathrm{pc}^{-2}$ in the SMC, corresponding to $A_{\mathrm{V}} \simeq 0.4$ and 0.2 , respectively. Thus, it appears that the "CO boundary" occurs at the same depth or extinction as the $\mathrm{HI}-\mathrm{H}_{2}$ boundary on average. This is in disagreement with theoretical models (Wolfire et al. 2010; Glover \& Mac Low 2011), which predict that $\mathrm{CO}$ emission should only appear for $A_{\mathrm{V}} \geqslant 1$. Understanding this difference will require more modeling work. On the one hand, it is possible that the mixing of different gas components within the beam may play a role. At $10-50$ pc resolution, a beam may contain both diffuse and dense gas. It is therefore possible that the $\mathrm{CO}$ emission arises from dense regions 

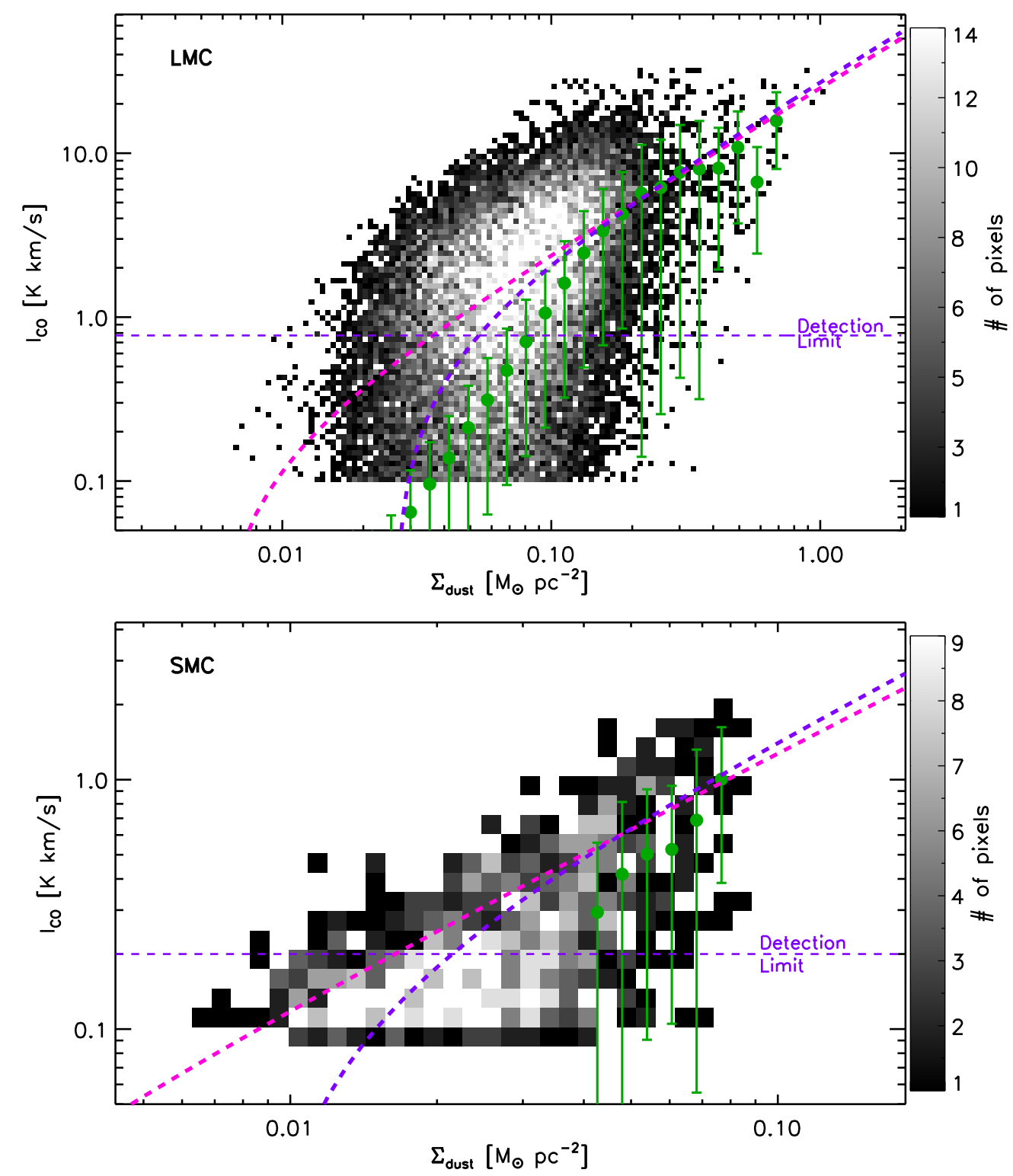

Figure 4. Pixel-to-pixel correlation between dust and CO emission in the LMC (top) and SMC (bottom). The grayscale shows the density of pixels, while the green circles correspond to the binned mean. The sensitivity limit, which corresponds to the $3 \sigma_{\mathrm{CO}}$ noise level in the SMC, and to the detection limit of the masking algorithm in the LMC, is indicated by the horizontal dashed purple line. The slope of the dust-CO relation, $\delta^{\mathrm{CO}}$, is shown as pink (linear fit) and purple (bisector fit) dashed lines. (A color version of this figure is available in the online journal.)

$\left(A_{\mathrm{V}}>1, \Sigma_{\text {dust }}>0.1 M_{\odot} \mathrm{pc}^{-2}\right)$ much smaller than the beam, in which the average surface density would be diluted to values $\left(A_{\mathrm{V}}<1\right)$ by the presence of diffuse gas. On the other hand, significant $\mathrm{CO}$ emission $\left(I_{\mathrm{CO}}>1 \mathrm{~K} \mathrm{~km} \mathrm{~s}^{-1}\right)$ is detected at $A_{\mathrm{V}} \sim$ 0.3 in the MW (Liszt \& Pety 2012) at 20" resolution. Therefore, numerical models may not be accurately accounting for "diffuse" CO emission.

\section{RELATION BETWEEN DUST AND TOTAL GAS}

We now combine the ionized, atomic, and molecular gas component to investigate the relation between dust and total gas surface densities (Figures 5 and 6). As a first step, we assume fiducial values of $X_{\mathrm{CO}}^{\mathrm{fid}}=1 \times 10^{21} \mathrm{~cm}^{-2} \mathrm{~K}^{-1} \mathrm{~km}^{-1} \mathrm{~s}$ in the SMC and $X_{\mathrm{CO}}^{\mathrm{fid}}=2 \times 10^{20} \mathrm{~cm}^{-2} \mathrm{~K}^{-1} \mathrm{~km}^{-1} \mathrm{~s}$ in the LMC, as justified in Section 2.3.2. We separate different surface density regimes. Pixels with low dust surface densities $\left(\Sigma_{\text {dust }} \leqslant\right.$
$\left.\Sigma_{d}^{\text {dif }}\right)$ and no significant $\mathrm{CO}$ emission are grouped under the "diffuse atomic phase" (red points in Figures 5 and 6). The molecular phase $\left(\Sigma_{\text {dust }} \geqslant \Sigma_{d}^{\text {dif }}\right)$ is separated into two groups. Pixels with $\Sigma_{\text {dust }} \geqslant \Sigma_{d}^{\text {dif }}$ and no significant $\mathrm{CO}$ emission are grouped under the "translucent molecular phase" (blue circles). These sight-lines contain a significant fraction of molecular gas, which cannot be traced since there is no spatially coincident $\mathrm{CO}$ emission. Therefore, we know we cannot account for the total gas mass in those regions. Pixels of the molecular phase with significant $\mathrm{CO}$ emission are grouped under the "dense" phase (green circles). The different groups of pixels are mapped in Figure 7.

The slope of the dust-total gas relation in the diffuse atomic phases has been calculated in Section 3, and is overlaid in Figures 5 and 6. The slope of the dust-gas relation for the diffuse atomic phase corresponds to the diffuse ISM GDR (red points). The dust-gas slope in the translucent phase does not provide any 

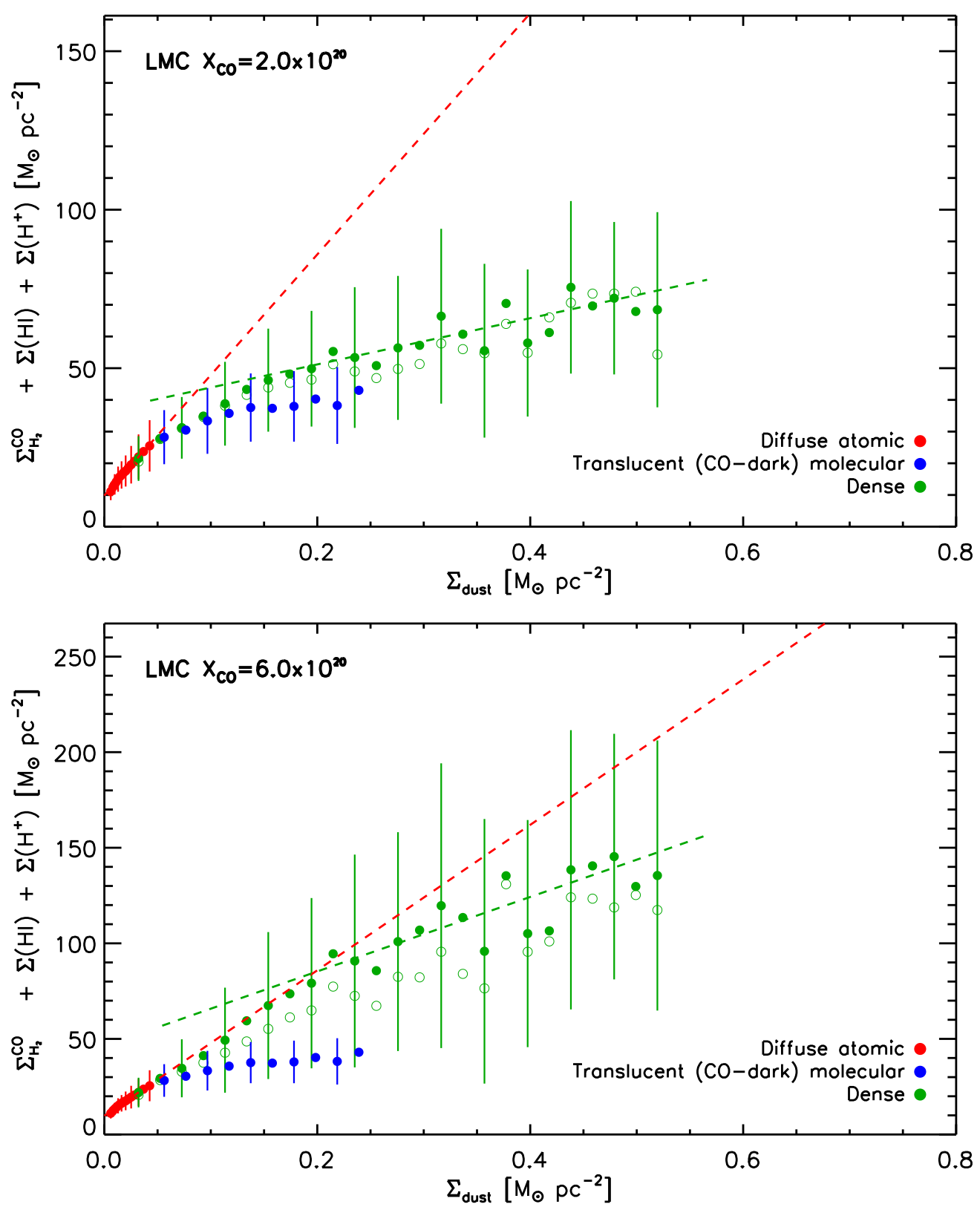

Figure 5. Pixel-to-pixel correlation (binned) between dust and total gas $\left(\mathrm{H} \mathrm{I}_{\mathrm{I}} \mathrm{H}_{2}, \mathrm{H}^{+}\right)$surface densities in the LMC. In the top panel, we assume our fiducial $X_{\mathrm{CO}}^{\mathrm{fid}}$ values for the $X$ factor $\left(2 \times 10^{20} \mathrm{~cm}^{-2} \mathrm{~K}^{-1} \mathrm{~km}^{-1} \mathrm{~s}\right)$. In the bottom panel, we assume our $X_{\mathrm{CO}}^{\text {max }}$ values $\left(6 \times 10^{20} \mathrm{~cm}^{-2} \mathrm{~K}^{-1} \mathrm{~km}^{-1} \mathrm{~s}\right)$. The red, blue, and green colors correspond to three different groups of pixels: black points correspond to the diffuse atomic phase and include pixels with $\Sigma_{\text {dust }} \leqslant \Sigma_{d}^{\text {dif }}$ and no detectable CO emission. Blue points correspond to the translucent phase, where a significant fraction of the total gas is most likely molecular, but is not traced by CO, and include pixels with $\Sigma_{\text {dust }} \geqslant \Sigma_{d}^{\text {dif }}$ and no $\mathrm{CO}$ emission. Green points are associated with "dense" pixels with CO emission above the sensitivity limit in the integrated intensity, determined by the masking algorithm. Filled circles show the binned mean, while empty circles show the binned median. The best-fit values of the slopes of the dust-total gas relation in the different groups of pixels are indicated in the legend and overlaid as dashed lines.

(A color version of this figure is available in the online journal.)

constraints on the GDR since the gas surface density is severely underestimated.

We must now calculate the slope of the dust-total gas relation in dense regions, including contributions from both atomic and molecular gas. In the SMC, Figure 6 shows that the relation between dust and gas in the dense phase is approximately linear. We fit a linear function to the pixel distribution with significant $\mathrm{CO}$ emission (and with a dust surface density with $\mathrm{S} / \mathrm{N} \geqslant 2$ ) using a $\chi^{2}$ minimization, accounting for proper measurement errors in the fit, using the uncertainties on the $\mathrm{H}$ I and $\mathrm{H} \alpha$ surface densities and CO integrated intensities listed in Section 2. We take as the $\mathrm{CO}$ detection limit the $3 \sigma_{\mathrm{CO}}$ noise level in the
SMC and find a slope $\delta^{\text {dense }}$ reported in Table 1. In the LMC, the dust-gas relation in the dense phase (CO detected) is not represented well with a linear function, i.e., the slope is not constant across the surface density range. In this case, therefore, we approximate this relation by two linear functions (i.e., a broken linear function), similar to Equation (3). We fit the dust-total gas pixel distribution with significant $\mathrm{CO}$ emission (and with a dust surface density with $\mathrm{S} / \mathrm{N} \geqslant 2$ ) to this two-piece linear function, accounting for proper measurement errors in the fit, again using the uncertainties on the $\mathrm{HI}$ and $\mathrm{H} \alpha$ surface densities and CO integrated intensities listed in Section 2. We take as the CO detection limit the detection limit in the $\mathrm{CO}$ 

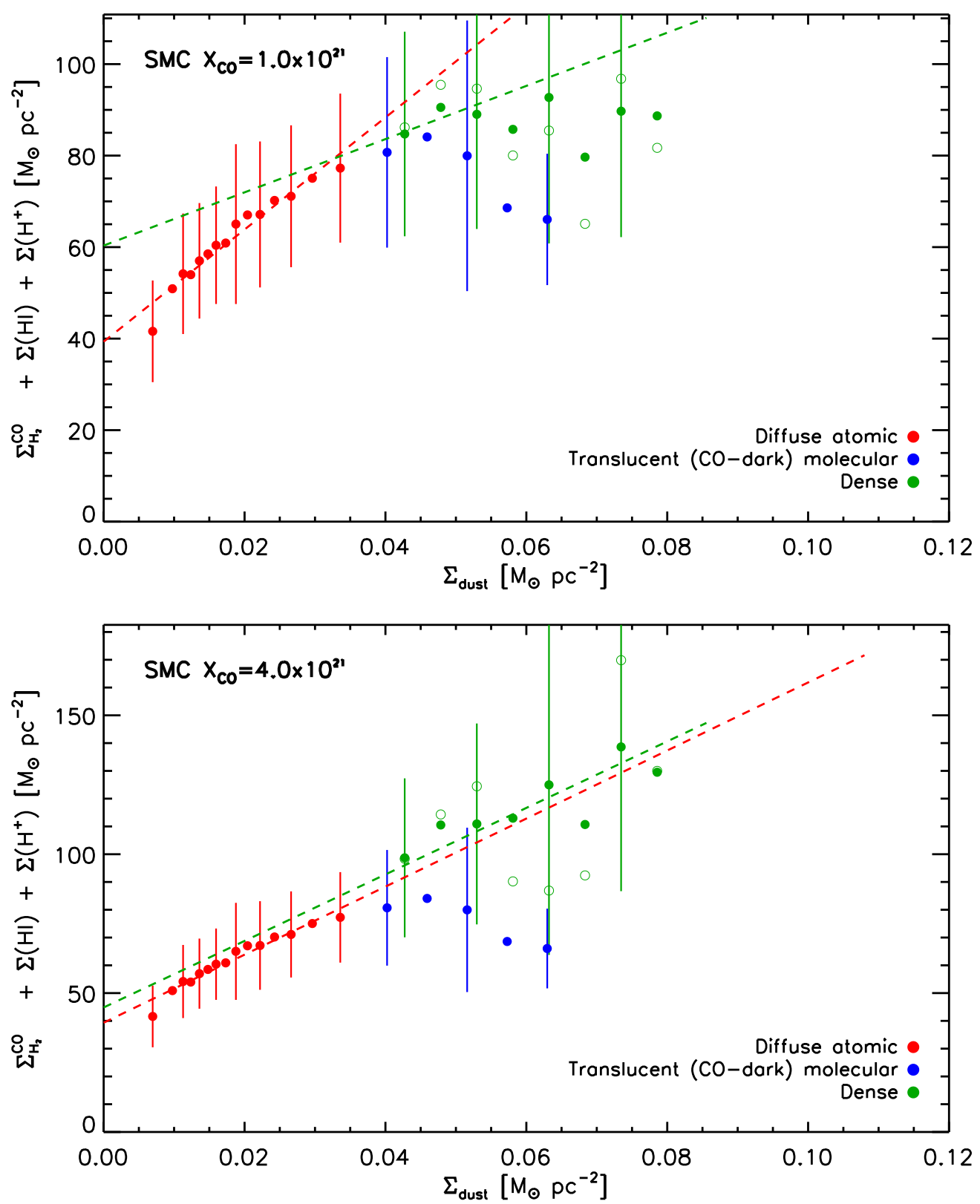

Figure 6. Pixel-to-pixel correlation (binned) between dust and total gas $\left(\mathrm{H} \mathrm{I}_{\mathrm{I}} \mathrm{H}_{2}, \mathrm{H}^{+}\right)$surface densities in SMC. In the top panel, we assume our fiducial $X_{\mathrm{CO}}^{\mathrm{fid}}$ values for the $X$ factor $\left(1 \times 10^{21} \mathrm{~cm}^{-2} \mathrm{~K}^{-1} \mathrm{~km}^{-1} \mathrm{~s}\right)$. In the bottom panel, we assume our $X_{\mathrm{CO}}^{\max }$ values $\left(4 \times 10^{21} \mathrm{~cm}^{-2} \mathrm{~K}^{-1} \mathrm{~km}^{-1} \mathrm{~s}\right)$. The red, blue, and green colors correspond to three different groups of pixels: black points correspond to the diffuse atomic phase and include pixels with $\Sigma_{\text {dust }} \leqslant \Sigma_{d}^{\text {dif }}$ and no detectable CO emission. Blue points correspond to the translucent phase, where a significant fraction of the total gas is most likely molecular, but is not traced by CO, and include pixels with $\Sigma_{\text {dust }} \geqslant \Sigma_{d}^{\text {dif }}$ and no $\mathrm{CO}$ emission. Green points are associated with "dense" pixels with CO emission above the CO sensitivity limit $\left(3 \sigma_{\mathrm{CO}}\right)$. Filled circles show the binned mean, empty circles show the binned median. The best-fit values of the slopes of the dust-total gas relation in the different groups of pixels are indicated in the legend and overlaid as dashed lines.

(A color version of this figure is available in the online journal.)

integrated intensity given by the masking algorithm in the LMC. We thus obtain a range of dust-total gas slopes in the dense ISM, $\delta_{\max }^{\text {dense }}-\delta_{\min }^{\text {dense }}$, applicable from low to high surface densities. The resulting values are listed in Table 1 . With our fiducial $X_{\mathrm{CO}}^{\text {fid }}$ values, the difference between GDR ${ }^{\text {dif }}$ and $\delta_{\min }^{\text {dense }}$ represents a factor $\geqslant 4$ decrease in the dust-gas slope between the diffuse and the densest ISM.

As for the dust-H I relation, there is a systematic uncertainty associated with the fitting method. In Appendix B, we apply different fitting procedures to the dust-total gas relation in the dense phase to gauge the range of dense dust-gas slopes. Assuming our fiducial value of $X_{\mathrm{CO}}^{\text {fid }}$, we find $\delta_{\min }^{\text {dense }}$ in the range
$70-130$ in the LMC, and $-240-580$ in the SMC. The decrease of the dust-gas slope from the diffuse to the dense ISM appears robust against the choice of fitting method. In the SMC, we note that a negative slope (or 0 within the errors) is not physical, and so it is very likely that our fiducial value of $X_{\mathrm{CO}}$ based on the Bolatto et al. (2013) prescription is too low.

\subsection{Interpretation of $\delta^{\text {dense }}$}

In principle, the derivative or slope of the dust-total gas relation should correspond to the GDR. However, in the dense phase, the slope of the observed dust-total gas relation can differ significantly from the true dense GDR due to the large 

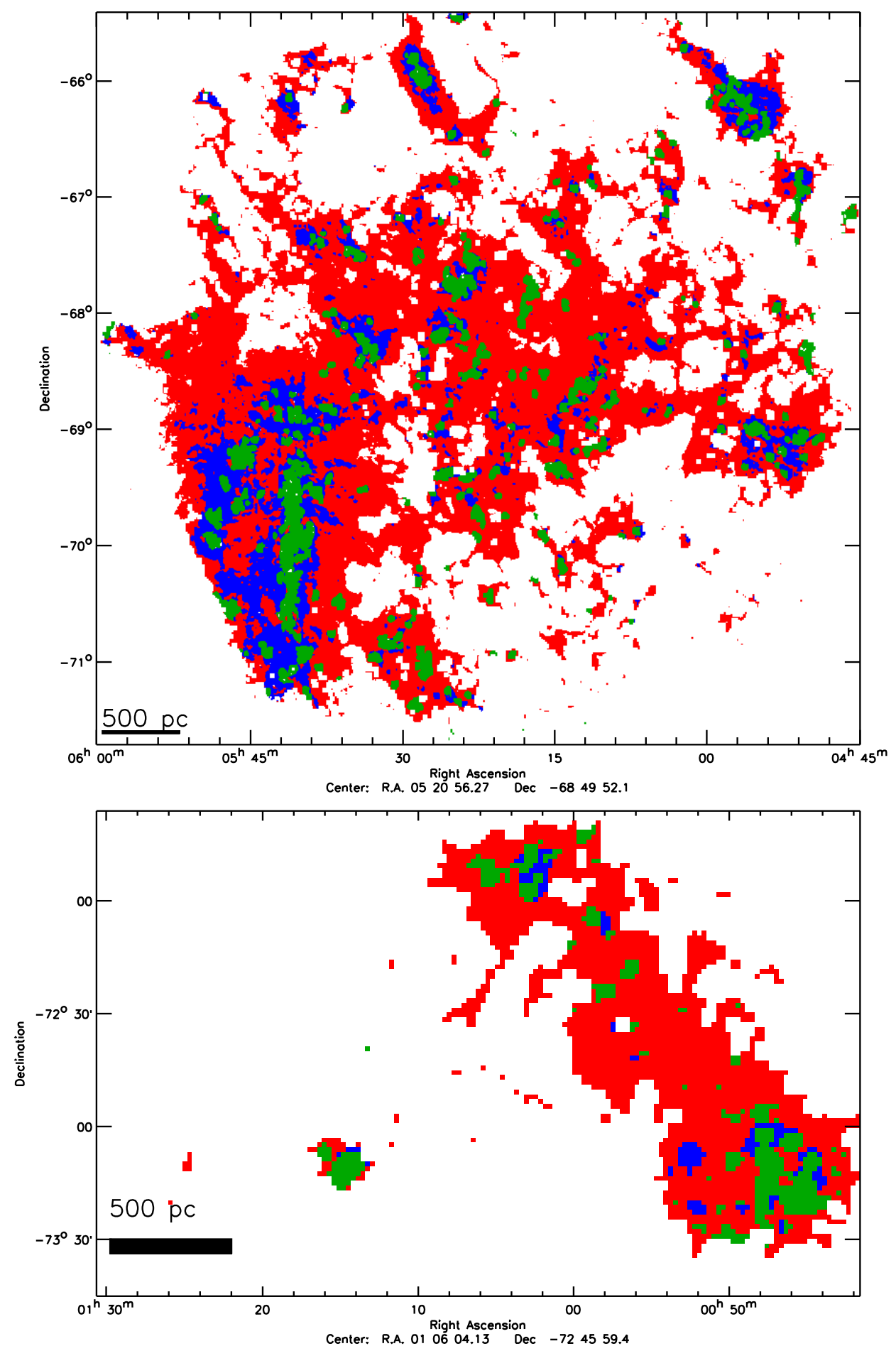

Figure 7. Map of the different phases in the LMC (top) and SMC (bottom). The red, blue, and green colors correspond to three different groups of pixels: red points correspond to the diffuse atomic phase and include pixels with $\Sigma_{\text {dust }} \leqslant \Sigma_{d}^{\text {dif }}$ and no detectable CO emission (below the $3 \sigma_{\mathrm{CO}}$ sensitivity limit in the SMC, below the sensitivity limit given by the masking algorithm in the LMC). Blue points correspond to the translucent molecular phase, where a significant fraction of the total gas is most likely molecular, but is not traced by $\mathrm{CO}$, and include pixels with $\Sigma_{\text {dust }} \geqslant \Sigma_{d}^{\text {dif }}$ and no CO emission. Green points are associated with "dense" pixels with CO emission above the $\mathrm{CO}$ sensitivity limit.

(A color version of this figure is available in the online journal.)

systematic uncertainties and degeneracies in the dust and gas surface density measurements. The dust surface density is potentially affected by emissivity variations incurred by dust grain coagulation in molecular clouds. Coagulation would cause the FIR emissivity of dust grains to increase with density. Since a constant emissivity is assumed in the dust mass derivation, this effect would lead to an overestimation of the dust surface density in the dense ISM, and produce an apparent decrease in the dust-gas slope with increasing surface density. This apparent decrease would be degenerate with true GDR (dust abundance) variations.

Additionally, gas surface densities derived from $\mathrm{H}_{\mathrm{I}} 21 \mathrm{~cm}$ and $\mathrm{CO}$ rotational emission may be underestimated due to the presence of optically thick H I (Stanimirovic et al. 1999; 
Dickey et al. 2000) and CO-dark $\mathrm{H}_{2}$ (Israel 1997; Leroy et al. 2007, 2009; Bolatto et al. 2011) in the translucent envelopes of molecular clouds. The presence of CO-dark $\mathrm{H}_{2}$ translates into a large systematic uncertainty in the CO-to- $\mathrm{H}_{2}$ conversion factor, $X_{\mathrm{CO}} \cdot X_{\mathrm{CO}}$ encompasses the effects of metallicity, chemistry, radiative transfer, dynamics, and geometry, and is too complex to model accurately. Observational constraints on $X_{\mathrm{CO}}$ also cover an order of magnitude range. With our initial "educated guess" of $X_{\mathrm{CO}}$, we found a significant decrease (factor $\geqslant 4$ ) in the dust-gas slope between the diffuse and dense ISM. But we will see in the next section that true GDR variations between ISM phases can be degenerate with the effects of CO-dark $\mathrm{H}_{2}$ in the beam of the $\mathrm{CO}$ observations. Without tight constraints on the right value of $X_{\mathrm{CO}}$ to use for a given set of environmental parameters, we cannot unambiguously constrain dust abundance variations between the diffuse and dense ISM.

\subsection{Effects of Different $X_{C O}$ Values}

Obviously the dense dust-gas slopes heavily depend on the assumed $X_{\mathrm{CO}}$. Therefore, we have performed a similar analysis for a range of $X_{\mathrm{CO}}$ values. Not surprisingly, we have found that $\delta^{\text {dense }}$ scales linearly with $X_{\mathrm{CO}}$, specifically,

$$
\delta_{\min }^{\text {dense }}=26.0+2.72 \times 10^{-19} X_{\mathrm{CO}}
$$

in the $\mathrm{LMC}$, and

$$
\delta^{\text {dense }}=376+2.05 \times 10^{-19} X_{\mathrm{CO}}
$$

in the SMC.

As a result, it is possible to obtain a nearly constant dust-total gas slope from the diffuse to dense ISM by choosing a higher $X_{\mathrm{CO}}$ than our fiducial values. In particular, for $X_{\mathrm{CO}}^{\max }=6 \times$ $10^{20} \mathrm{~cm}^{-2} \mathrm{~K}^{-1} \mathrm{~km}^{-1} \mathrm{~s}$ in the LMC and $X_{\mathrm{CO}}^{\max }=4 \times$ $10^{21} \mathrm{~cm}^{-2} \mathrm{~K}^{-1} \mathrm{~km}^{-1} \mathrm{~s}$ in the SMC, the slope of the dust-gas correlation remains constant within the errors across most of the surface density range (bottom panels of Figures 5 and 6). Values of $\delta^{\text {dense }}\left(\delta_{\mathrm{min}}^{\text {dense }}\right.$ in the LMC) obtained with $X_{\mathrm{CO}}^{\mathrm{max}}$ are reported in Table 1.

For values of $X_{\mathrm{CO}} \geqslant X_{\mathrm{CO}}^{\max }$, the slope of the dust-gas correlation is higher in the dense ISM than in the diffuse ISM, which is not physical. Physical mechanisms in the ISM that may affect the dust-gas slope, such as $\mathrm{CO}$-dark $\mathrm{H}_{2}$, dust coagulation, optically thick H I, and dust growth, all result in a decrease of the dust-gas slope with increasing surface density. Therefore, the values of $X_{\mathrm{CO}}^{\max }$ represent upper limits for $X_{\mathrm{CO}}$.

In the $\mathrm{SMC}$, this value of $X_{\mathrm{CO}}^{\max }$, which is within the range expected from Equation (2) and implies $\Sigma_{\mathrm{GMC}, 100}=0.6$, ensures that the dust-gas slope is constant across ISM phases. In the $\mathrm{LMC}$, this value of $X_{\mathrm{CO}}^{\mathrm{max}}$, which corresponds to $\Sigma_{\mathrm{GMC}, 100}=0.5$, yields a constant dust-gas slope up to $\Sigma_{\text {dust }}=0.4 M_{\odot} \mathrm{pc}^{-2}$, above which the dust-gas-relation becomes shallower, with a slope of $\delta_{\min }^{\text {dense }} \simeq 190$. The dust-gas slope at the highest surface densities of the LMC is thus a factor $\sim 2$ lower than in the diffuse ISM, even after accounting for the presence of CO-dark $\mathrm{H}_{2}$ via a higher than Galactic $X$ factor.

\subsection{Evidence of Dust Growth in LMC Molecular Clouds?}

In the LMC, there is still a flattening of the dust-gas relation for $\Sigma_{\text {dust }} \geqslant 0.4 M_{\odot} \mathrm{pc}^{-2}$ when $X_{\mathrm{CO}}^{\max }$ is assumed. The systematic uncertainties on the dust-gas slopes are large, and include two main sources: the uncertainty on the dust emission, $\kappa_{160}$ and the uncertainty due to the fitting technique (see Appendices A and B and Section 3.1). On the one hand, the uncertainty on the dust emissivity $\kappa_{160}$ applies to all phases in a similar manner, and therefore cancels out when examining variations of the dust-gas slope. On the other hand, the uncertainty due to the fitting technique differs between the diffuse and dense phases. Nonetheless, the decrease of the dust-gas slope from the diffuse to the dense phase of the LMC appears robust against the fitting method. Values of $\delta_{\min }^{\text {dense }}$ are derived with $X_{\mathrm{CO}}^{\max }$ using different fitting techniques in Appendix B, where we find that $\delta_{\min }^{\text {dense in }}$ the LMC is in the range 180-330. For comparison, different fits to the dust-gas relation in the diffuse atomic ISM yield a range $\mathrm{GDR}^{\text {dif }}=380-540$.

If this decrease of the dust-gas slope from the diffuse to dense ISM were due to $X_{\mathrm{CO}}$ variations, it would mean that $X_{\mathrm{CO}}$ would increase with increasing surface density. However, we expect the opposite at this metallicity: $X_{\mathrm{CO}}$ should decrease with increasing surface density (Shetty et al. 2011).

Two likely explanations for the factor $\sim 2$ decrease of the dust-gas slope between diffuse and dense ISM in the LMC are dust grain coagulation and/or accretion of gas-phase metals onto dust grains (true GDR variations). With grain coagulation, the GDR would stay constant and equal GDR ${ }^{\text {dif }}$, but the FIR emissivity of dust grains would increase in molecular clouds. With grain accretion, the dust abundance and therefore dustto-gas ratio would increase from the diffuse to the dense ISM. In this case, the dense GDR would be equal to $\delta^{\text {dense }}$. Coagulation and accretion are degenerate and are expected to have similar effects on the dust-gas slope, which can be interpreted as an apparent GDR. Both processes would occur in similar density ranges, and incur apparent variations in the GDR with similar magnitudes. In a future paper, we introduce simple theoretical modeling of coagulation and accretion to constrain the magnitude of these effects, and determine how much each effect contributes to the decrease of the dust-gas slope between the diffuse and dense ISM. Another possible mechanism leading to a decrease in the dust-gas slope between the diffuse and dense phases is grain clustering due to turbulence (Hopkins 2014).

\subsection{Mass of the $\mathrm{CO}$-dark $\mathrm{H}_{2}$ Component in the Translucent Phase}

Assuming that the true translucent GDR is the same as in the diffuse ISM, the total $\mathrm{H}_{2}$ mass associated with the $\mathrm{CO}$ dark translucent component can be computed as $M\left(\mathrm{H}_{2}^{\mathrm{CO}-\text { dark }}\right)=$ $\mathrm{GDR}^{\text {dif }} \times M_{\text {dust }}^{\text {trans }}-M\left(\mathrm{H}_{\mathrm{I}}{ }^{\text {trans }}\right)$. We find $M\left(\mathrm{H}_{2}^{\mathrm{CO}-\text { dark }}\right)=1.0 \times$ $10^{7} M_{\odot}$ in the LMC (4\% of the $\mathrm{H}$ I mass), and $M\left(\mathrm{H}_{2}^{\mathrm{CO}-\text { dark }}\right)=$ $1.3 \times 10^{6} M_{\odot}$ in the SMC ( $1 \%$ of the H i mass). The CO-dark $\mathrm{H}_{2}$ masses represent $100 \%-30 \%$ and $40 \%-10 \%$ of the CObright $\mathrm{H}_{2}$ mass in the LMC and SMC, with $X_{\mathrm{CO}}$ in the range $X_{\mathrm{CO}}^{\mathrm{fid}}-X_{\mathrm{CO}}^{\mathrm{max}}$. We note that, since this mass of CO-dark gas is computed in pixels with no $\mathrm{CO}$ detection, it does not include $\mathrm{CO}$ dark molecular gas located in the beam of the $\mathrm{CO}$ observations accounted for by the use of a higher than galactic $X_{\mathrm{CO}}$.

\section{DISCUSSION}

\subsection{Comparison to Depletions and UV Measurements}

Since the systematic uncertainty on our GDR measurements is large, it is useful to compare them to independent measurements using UV absorption spectroscopy, which have a lower systematic uncertainty. Welty et al. (2012) combined column densities of $\mathrm{HI}$ and $\mathrm{H}_{2}$ toward LMC and SMC sight-lines from archival Hubble Space Telescope and FUSE spectra with 

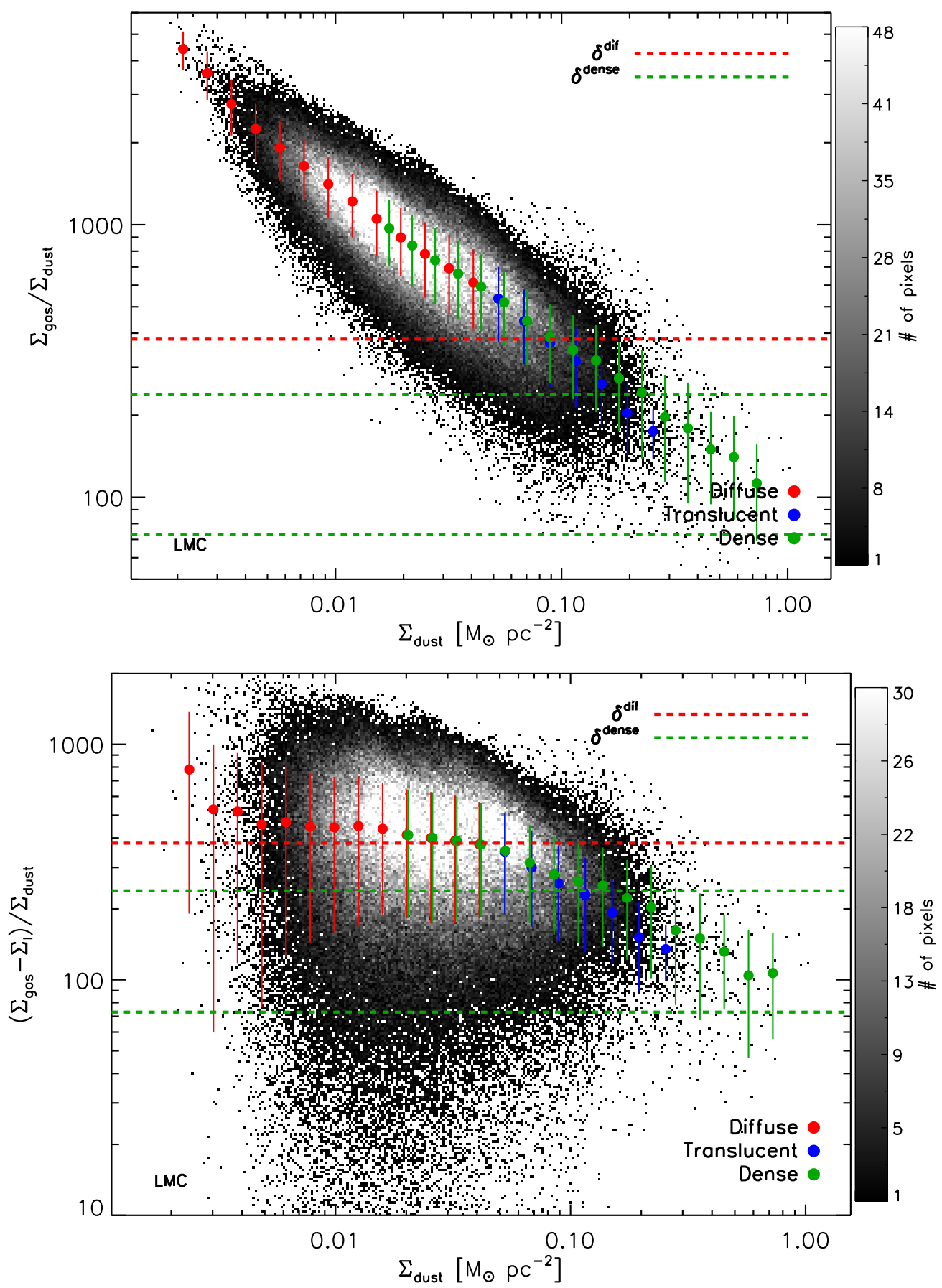

Figure 8. Pixel-to-pixel relation between the surface density ratio, which can be interpreted as an apparent gas-to-dust ratio, and the dust surface density in the LMC. Our fiducial $X_{\mathrm{CO}}$ values are assumed. In the top panels, the surface density ratio is computed as $\Sigma_{\text {gas }} / \Sigma_{\text {dust }}$, while the bottom panels show the surface density ratio after the gas pedestal $\Sigma_{I}$ is subtracted from the gas, i.e., $\left(\Sigma_{\text {gas }}-\Sigma_{I}\right) / \Sigma_{\text {dust }}$. Subtracting the gas pedestal is roughly equivalent to computing the surface density ratio in the volume where FIR emission is detected. The contours indicate the distribution of GDR and $\Sigma_{\text {dust }}$ measurements for individual pixels, while the filled circles show the binned mean in each $\Sigma_{\text {dust }}$ bin. The red, blue, and green points correspond to the diffuse, translucent and dense phases. For comparison, the red and green dashed lines correspond to the dust-gas slope values GDR ${ }^{\text {dif }}$ and $\delta_{\max }^{\text {dense }}-\delta_{\min }^{\text {dense }}$.

(A color version of this figure is available in the online journal.)

extinction information $(E(B-V))$, and derived GDRs of $3 \pm$ 1.5 times the MW GDR in the LMC, and $6 \pm 3$ times the MW GDR in the SMC. This corresponds to GDRs of $450 \pm 220$ in the $\mathrm{LMC}$, and $900 \pm 450$ in the SMC, which is consistent with the values derived here.

Additionally, Jenkins (2009) derived the MW depletions of metals, which correspond to the fraction of metals locked up in dust grains, as a function of density. By combining these measurements with Magellanic Cloud abundances taken from
Russell \& Dopita (1992), we can sum up the mass of metals in dust grains and estimate the GDR as a function of density, in the diffuse ISM and in clouds. We can also compute a mathematical lower limit on the GDR by assuming that all metals are locked up in dust grains. The resulting diffuse and cloud GDR values are listed in Table 2.

This calculation assumes that the relation between density and the depletion patterns is the same in the MW and in the Magellanic Clouds. We note that there may be significant 

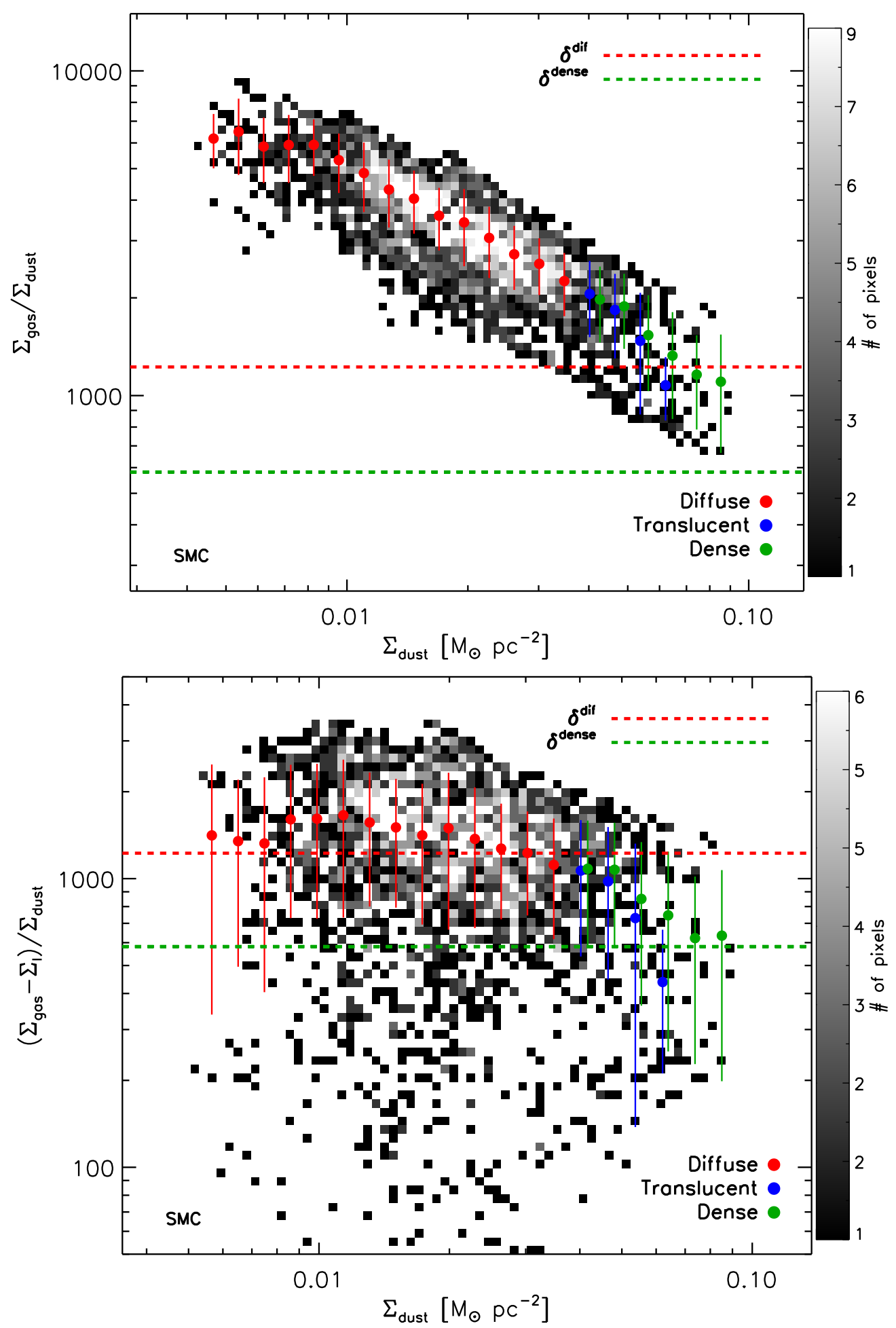

Figure 9. Pixel-to-pixel relation between the surface density ratio, which can be interpreted as an apparent gas-to-dust ratio, and the dust surface density for the SMC. Our fiducial $X_{\mathrm{CO}}$ values are assumed. In the top panels, the surface density ratio is computed as $\Sigma_{\text {gas }} / \Sigma_{\text {dust }}$, while the bottom panels show the surface density ratio after the gas pedestal $\Sigma_{I}$ is subtracted from the gas, i.e., $\left(\Sigma_{\text {gas }}-\Sigma_{I}\right) / \Sigma_{\text {dust }}$. Subtracting the gas pedestal is roughy equivalent to computing the surface density ratio in the volume where FIR emission is detected. The contours indicate the distribution of GDR and $\Sigma_{\text {dust }}$ measurements for individual pixels, while the filled circles show the binned mean in each $\Sigma_{\text {dust }}$ bin. The red, blue, and green points correspond to the diffuse, translucent and dense phases. For comparison, the red and green dashed lines correspond to the dust-gas slope values GDR ${ }^{\text {dif }}$ and $\delta_{\max }^{\text {dense }}-\delta_{\min }^{\text {dense }}$.

(A color version of this figure is available in the online journal.)

differences between depletion patterns in the MW and dwarf galaxies (Sofia et al. 2006). For example, depletion fractions may be lower at low metallicity (K. Tchernyshyov et al., in preparation), leading to a non-linear relation between GDR and metallicity. Correspondingly, Rémy-Ruyer et al. (2014) find a power-law relation between GDR and metallicity based on a sample of dwarf galaxies with metallicities $12+\log (\mathrm{O} / \mathrm{H}) \sim$ $7-9$. Such variations of the depletion fractions with metallicity 
Table 2

Gas-to-dust Ratios Predicted from Elemental Abundances and Depletions

\begin{tabular}{lccc}
\hline \hline GDR & MW & LMC & SMC \\
\hline GDR $_{\text {dif }}^{\text {dep }}$ & $322 \pm 150$ & $428 \pm 200$ & $1260 \pm 620$ \\
GDR $_{\text {cloud }}^{\text {dep }}$ & $126 \pm 10$ & $185 \pm 25$ & $528 \pm 72$ \\
GDR $_{\text {min }}^{\text {dep }}$ & 68.1 & $117 \pm 13$ & $298 \pm 45$ \\
\hline
\end{tabular}

\section{Notes.}

${ }^{a} \mathrm{GDR}_{\mathrm{dif}}^{\mathrm{dep}}$ is the GDR obtained from MW depletions and Magellanic Cloud abundances in the diffuse ISM.

b $\mathrm{GDR}_{\text {cloud }}^{\text {dep }}$ is the GDR obtained from MW depletions and Magellanic Cloud abundances in the translucent to dense ISM.

${ }^{c} \mathrm{GDR}_{\mathrm{min}}^{\mathrm{dep}}$ is the mathematical lower limit on the GDR obtained by assuming Magellanic Cloud abundances and depletion fractions of 1 for all elements (all metals locked up in dust).

may be explained by the lower filling factor of the dense ISM, and the subsequently enhanced dust destruction rate. Hence, the GDR values predicted from Magellanic abundances and MW depletion fractions represent a lower limit.

The diffuse atomic GDRs and dense dust-gas slopes derived here are consistent within the errors with values derived from depletion fractions and abundances. In addition, depletion measurements do suggest that gas-phase metals may accrete onto dust grains in molecular clouds, thus changing the GDR in the dense phase. While our emission-based measurements are affected by degeneracies between true GDR variations, $\mathrm{CO}$ dark $\mathrm{H}_{2}$ and dust grain coagulation, the comparison to depletion fractions supports the idea that dust grains grow in the dense ISM. Thus, the variations of the dust-gas slopes are probably not just caused by the presence of CO-dark $\mathrm{H}_{2}$.

\subsection{Gas-to-dust Ratio Measured as a Ratio of Surface Densities}

It is useful to derive maps of the GDR, for instance, to examine the relation between its spatial variations and environmental factors like star formation rate, radiation field, dynamical environment etc. Such maps can only be obtained by computing the GDR as a ratio of surface densities (not a slope). Because we know that such ratio maps are biased due to the presence of CO-dark $\mathrm{H}_{2}$, and systematic uncertainties on the dust surface densities due to poor constraints on the FIR emissivity and its variations with density, we refer to such maps as apparent GDR maps.

We have shown that the dust-gas correlation is not linear. At dust surface densities for which FIR emission is not detected, the dust-gas slope is likely a factor five higher than in FIRbright diffuse regions. At high surface densities, in the dense ISM, we have shown that the dust-gas slope is shallower than in the diffuse ISM, by a factor at least $\sim 2$ in the LMC. As a result, taking the ratio of gas and dust surface densities results in a quantity that is not well defined, and includes different ISM components along the line-of-sight. The top panels of Figures 8 and 9 show the relation between $\Sigma_{\text {gas }} / \Sigma_{\text {dust }}$ and $\Sigma_{\text {dust }}$. At low surface densities, this ratio is dominated by the FIR-faint gas component. As a result, $\Sigma_{\text {gas }} / \Sigma_{\text {dust }}$ values in the FIR-bright diffuse phase are much higher than the diffuse dust-gas slope $\mathrm{GDR}^{\text {dif }}$.

In the bottom panels, we show the ratio $\left(\Sigma_{\text {gas }}-\Sigma_{I}\right) / \Sigma_{\text {dust }}$ as a function of $\Sigma_{\text {dust }}$. In this case, the surface density ratio in the diffuse phase is compatible with the diffuse dust-gas slope. This is because subtracting the gas pedestal $\Sigma_{I}$ is roughly equivalent to computing the surface density ratio in the volume where FIR emission is detected.

Therefore, we favor the derivation of the GDR as a slope, which appears to be more robust and also allows us to statistically separate the different ISM phases.

\section{CONCLUSION}

Based on Herschel FIR, H I $21 \mathrm{~cm}, \mathrm{CO} J=1-0$, and $\mathrm{H} \alpha$ emission maps of the Magellanic Clouds, we investigate the relation between dust and gas surface densities at $10-50 \mathrm{pc}$ resolution. We find that the dust-H I relation is linear up to $\Sigma_{\text {dust }}=$ $0.05 M_{\odot} \mathrm{pc}^{-2}$ in the LMC and $\Sigma_{\text {dust }}=0.03 M_{\odot} \mathrm{pc}^{-2}$ in the SMC, corresponding to $A_{\mathrm{V}}=0.4$ and 0.2 , respectively. These surface densities correspond to the atomic-to-molecular transition, above which the dust-H I relation saturates at $\Sigma(\mathrm{HI})=$ $40 M_{\odot} \mathrm{pc}^{-2}$ in the LMC and $70 M_{\odot} \mathrm{pc}^{-2}$ in the SMC. These surface densities do not include the contribution of helium, nor do GDR and dust-gas slope values reported in this study.

Below those surface densities, the slope of the dustH I relation corresponds to the diffuse atomic GDR, which, in the LMC, we find to be $\mathrm{GDR}^{\text {dif }}=380-540$ depending on the fitting method, with a systematic uncertainty of $35 \%$ (and not including helium). In the SMC, we find GDR ${ }^{\text {dif }}=1200-2100$ with a systematic uncertainty of $35 \%$. Those values are in excellent agreement with observed elemental abundances and depletion patterns.

We find a pedestal in the dust-gas relation, which corresponds to $\mathrm{H}$ I surface densities of $9.8 M_{\odot} \mathrm{pc}^{-2}$ in the LMC, and $39 M_{\odot} \mathrm{pc}^{-2}$ in the SMC. This pedestal can be explained by the presence of a dust-poor gas component below the sensitivity limit of our FIR maps surrounding the FIR-bright regions, with GDR 5-10 times higher than in the FIR-bright diffuse atomic ISM.

In the dense ISM, where CO emission is detected, the dust-gas slope does not necessarily correspond to the GDR. The gas surface density estimates are affected by large systematic errors due to the poorly constrained $X_{\mathrm{CO}}$ factor. Additionally, coagulation may increase the FIR emissivity of dust grains in the molecular clouds. A constant emissivity (for lack of any other model or prior) is assumed in the dust surface density derivation. As a result, the effect of coagulation would be an overestimation of the dust surface density in the dense phase. These two effects both result in a decrease of the dust-gas slope from the diffuse to the dense ISM, and therefore are degenerate with true GDR variations. GDR variations may be incurred by accretion of gas-phase metals onto dust grains in the dense ISM, or turbulent clustering of dust grains in molecular clouds.

The dense ISM dust-gas slope depends on the assumed $X_{\mathrm{CO}}$ factor. We derive upper limits of $X_{\mathrm{CO}}^{\mathrm{max}}=6 \times$ $10^{20} \mathrm{~cm}^{-2} \mathrm{~K}^{-1} \mathrm{~km}^{-1} \mathrm{~s}$ in the LMC, and $X_{\mathrm{CO}}^{\max }=4 \times$ $10^{21} \mathrm{~cm}^{-2} \mathrm{~K}^{-1} \mathrm{~km}^{-1} \mathrm{~s}$ in the SMC, which is within the range expected from theoretical models and previous observations.

In the densest regions of the SMC, the dust-gas slope ranges from $\delta^{\text {dense }}=0-1900$ with $X_{\mathrm{CO}}=1-4 \times 10^{21} \mathrm{~cm}^{-2} \mathrm{~K}^{-1} \mathrm{~km}^{-1}$ $\mathrm{s}$ and depending on the fitting method, with a systematic uncertainty of $35 \%$ (not including helium). Assuming the maximum value of $X_{\mathrm{CO}}$ results in a constant dust-gas slope across ISM phases, while lower $X_{\mathrm{CO}}$ values result in a significant decrease of the dust-gas slope between the diffuse and dense ISM. Thus, variations of the dust-gas slope between diffuse and 

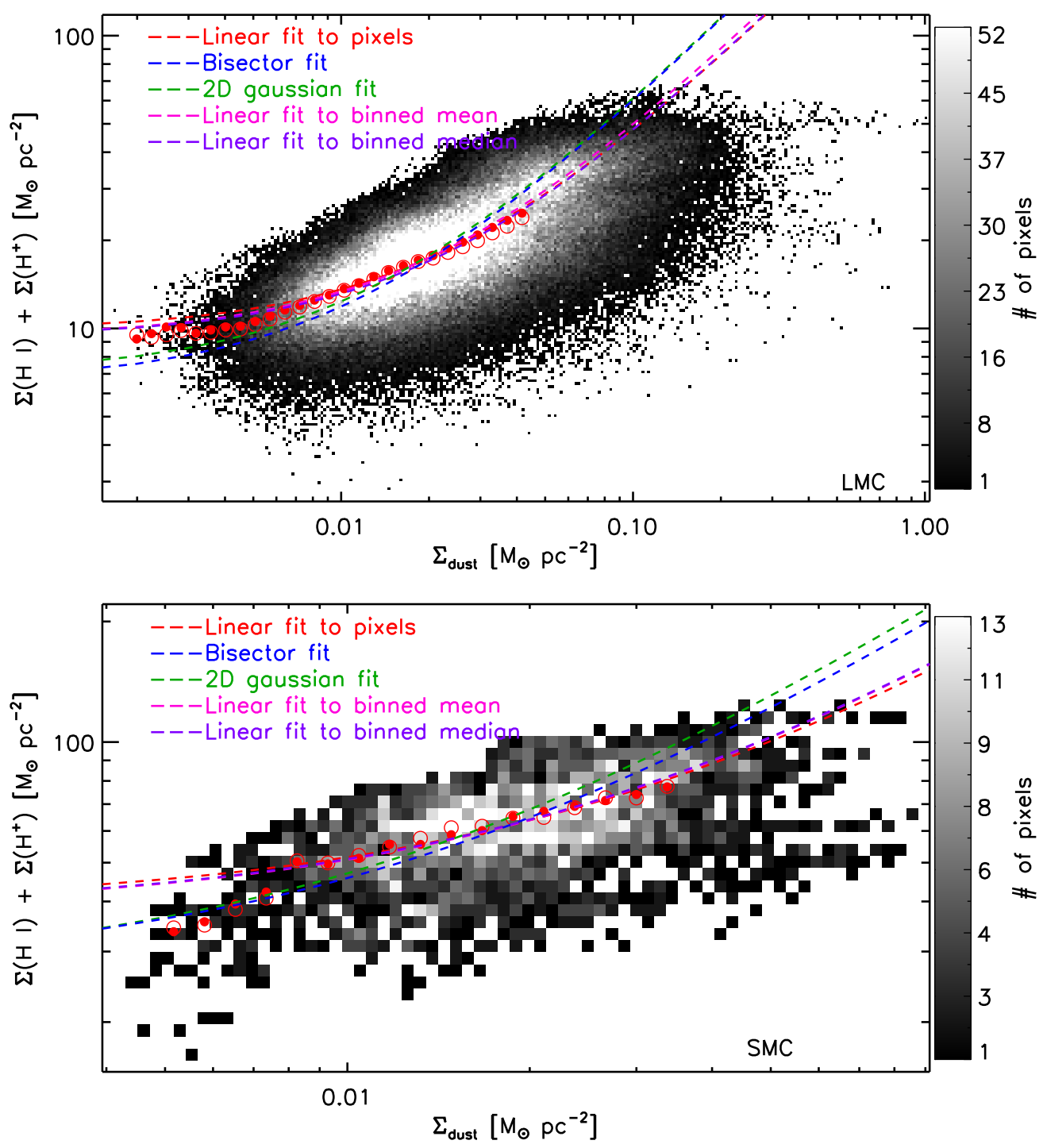

Figure 10. Pixel-to-pixel correlation between dust and atomic + ionized gas surface densities in the LMC (top) and SMC (bottom). The grayscale shows the density of points. The filled red circles show the binned mean atomic gas surface density in the diffuse atomic medium, while the empty red circles show the binned median. Over-plotted are different linear fits. Our fiducial method, which consists in performing a $\chi^{2}$ minimization of the pixels to a linear function, is shown as a red dashed line. We also performed a bisector fit (blue), a two-dimensional Gaussian fit to the $\Sigma_{\text {dust }}-\Sigma(\mathrm{HI}) \log -\log$ distribution (green), a linear fit to the binned mean (pink), and a linear fit to the binned median (purple). The resulting dust-gas slopes are summarized in Table 3.

(A color version of this figure is available in the online journal.)

Table 3

Parameters of the Dust-H I Relation Obtained with Different Fitting Methods (with the BEMBB Dust Model)

\begin{tabular}{lccrrr}
\hline \hline Galaxy & Dust Model & GDR $_{\text {gauss }}^{\text {dif }}{ }^{2}$ & GDR $_{\text {bisect }}^{\text {dif }}{ }^{2}$ & \multicolumn{1}{c}{ GDR $_{\text {mean }}^{\text {dif }}{ }^{b}$} & GDR $_{\text {med }}^{\text {dif }}$ c \\
\hline LMC & BEMBB & $540 \pm 40 .(-9.2 \%)$ & $540 \pm 4.3(-7.0 \%)$ & $409 \pm 2.9(-9.5 \%)$ & $390 \pm 3.1(-12 . \%)$ \\
SMC & BEMBB & $2100 \pm 3900(-55 \%)$ & $1900 \pm 200(-9.4 \%)$ & $1300 \pm 120(-17 \%)$ & $1300 \pm 130(-20 \%)$ \\
\hline
\end{tabular}

Notes. Values are given as value \pm random uncertainty (bias on the parameter recovery, obtained from output-input in a Monte-Carlo simulation). The systematic uncertainty on the dust-gas slopes is $35 \%$ (see Appendix A).

${ }^{a} \mathrm{GDR}_{\text {gauss }}^{\text {dif }}$ is the dust-atomic gas slope derived from a two-dimensional Gaussian fit to the dust-atomic gas $\log -\log$ distribution.

${ }^{\mathrm{b}} \mathrm{GDR}_{\text {mean }}^{\text {dif }}$ is the dust-atomic gas slope derived from a linear fit to the binned average.

${ }^{c} \mathrm{GDR}_{\text {med }}^{\text {dif }}$ is the dust-atomic gas slope derived from a linear fit to the binned median.

${ }^{\mathrm{d}} \mathrm{GDR}_{\mathrm{bisect}}^{\mathrm{dif}}$ is the dust-atomic gas slope derived from bisector fit to the dust-atomic gas distribution.

dense ISM are degenerate with the effects of CO-dark $\mathrm{H}_{2}$ via the choice of $X_{\mathrm{CO}}$ factor. Further modeling and observations are required to break this degeneracy.

In the LMC, even when accounting for CO-dark $\mathrm{H}_{2}$ by assuming the maximum value of $X_{\mathrm{CO}}$, the maximum dense ISM dust-gas slope we obtain is $\delta^{\text {dense }}=180-330$ depending on the fitting method, again with a systematic uncertainty of $35 \%$ (not including helium). Since the systematic uncertainty applies in the same way in all phases, the dust-gas slope is significantly lower (factor $\geqslant 2$ ) in the dense ISM compared to the diffuse 


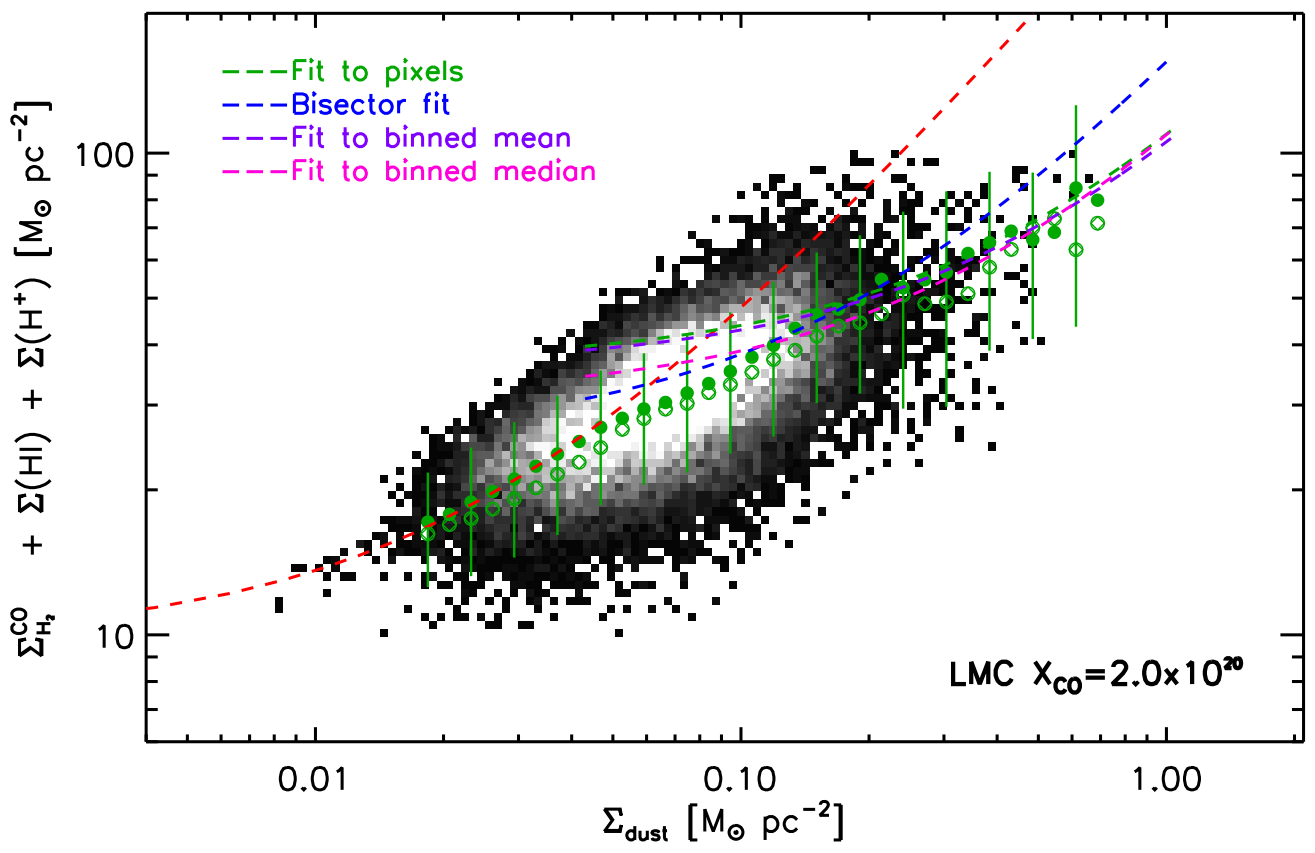

Figure 11. Pixel-to-pixel correlation between dust and total gas surface densities in the dense phase (CO detected) of the LMC, assuming values $X_{\mathrm{CO}}^{\text {fid }}$. The distribution, shown by the grayscale, only includes pixels with $\mathrm{CO}$ detections. The green filled and empty circles correspond to the binned mean and median (as in Figure 5). Overplotted are different linear fits. Our fiducial method, which consists in performing a $\chi^{2}$ minimization of the pixels to a linear function, is shown as a green dashed line. We also performed a bisector fit (blue), a linear fit to the binned mean (pink), and a linear fit to the binned median (purple). The resulting dust-gas slopes are summarized in Table 4.

(A color version of this figure is available in the online journal.)

Table 4

Parameters of the Dust-Total Gas Relation in the Dense phase Obtained with Different Fitting Methods (with the BEMBB Dust Model)

\begin{tabular}{lccccc}
\hline \hline Galaxy & Dust Model & $\begin{array}{c}X_{\mathrm{CO}} \\
\left(\mathrm{cm}^{-2} \mathrm{~K}^{-1} \mathrm{~km}^{-1} \mathrm{~s}\right)\end{array}$ & $\delta_{\text {min,bisect }}^{\text {dense }}$ & $\delta_{\text {min,mean }}^{\text {dense }}$ & $\delta_{\text {min,med }}^{\text {dense }}$ \\
& & $2 \times 10^{20}$ & $130 \pm 4.1(24 \%)$ & $70 \pm 14(0.9 \%)$ & $78 \pm 14(-9.6 \%)$ \\
\hline LMC & BEMBB & $6 \times 10^{20}$ & $330 \pm 20(-23 \%)$ & $180 \pm 38(-36 \%)$ & $190 \pm 47(-26 \%)$ \\
& BEMBB & $1 \times 10^{21}$ & $1100 \pm 41 .(-15 \%)$ & $8.3 \pm 260(910 \%)$ & $-240 \pm 360(-130 \%)$ \\
SMC & BEMBB & $4 \times 10^{21}$ & $1900 \pm 54(-26 \%)$ & $890 \pm 360(-36 \%)$ & $850 \pm 530(-16 \%)$
\end{tabular}

Notes. Values are given as value \pm systematic uncertainty \pm random uncertainty (bias on the parameter recovery, obtained from output-input in a Monte-Carlo simulation). The systematic uncertainty on the dust-gas slopes and on $\Sigma_{d}^{\text {dif }}$ is $35 \%$ (see Appendix A).

${ }^{a} \delta_{\text {bisect }}^{\text {dense, } i n}$ is the dust-total gas slope derived in the dense phase at the highest surface densities from a bisector fit to the $\Sigma_{\text {dust }}-\Sigma_{\text {gas }}$ distribution.

${ }^{\mathrm{b}} \delta_{\text {mean }}^{\text {dense, } m i n}$ is the dust-total gas slope derived in the dense phase at the highest surface densities from a linear fit to the binned mean.

${ }^{c} \delta_{\text {mean }}^{\text {dense }}{ }^{2 i n}$ is the dust-total gas slope derived in the dense phase at the highest surface densities from a linear fit to the binned median.

Table 5

Parameters of the Fits to the Dust-gas Relation (with the SMBB and TTMBB Dust Model)

\begin{tabular}{|c|c|c|c|c|c|c|}
\hline \multirow[t]{2}{*}{ Galaxy } & \multirow[t]{2}{*}{ Dust Model } & \multicolumn{3}{|c|}{ Diffuse ISM Parameters } & \multicolumn{2}{|c|}{ Dense Phase Parameters } \\
\hline & & $\mathrm{GDR}^{\mathrm{dif}}$ & $\begin{array}{c}\Sigma_{I}^{\mathrm{a}} \\
\left(M_{\odot} \mathrm{pc}^{-2}\right)\end{array}$ & $\begin{array}{c}\sum_{d}^{\mathrm{difb}} \\
\left(M_{\odot} \mathrm{pc}^{-2}\right)\end{array}$ & $\delta_{\min }^{\text {dense } c, d}$ & $\delta_{\min }^{\text {dense }}$ \\
\hline SMC & $\begin{array}{l}\text { SMBB } \\
\text { TTMBB }\end{array}$ & $\begin{array}{l}2300 \pm 180(-29 \%) \\
1200 \pm 25(-81 \%)\end{array}$ & $\begin{aligned} 32 . & \pm 1.9(26 \%) \\
30 & \pm 0.7(86 \%)\end{aligned}$ & $\begin{array}{l}0.02 \pm 0.004(9.9 \%) \\
0.03 \pm 0.03(70 \%)\end{array}$ & $\begin{array}{c}960 \pm 49(-7.1 \%) \\
3.8 \pm 11(470 \%)\end{array}$ & $\begin{aligned} 1900 & \pm 69(-21 \%) \\
94 & \pm 27(-23 \%)\end{aligned}$ \\
\hline
\end{tabular}

Notes. Values are given as value \pm random uncertainty (bias on the parameter recovery, obtained from output-input in a Monte-Carlo simulation). The systematic uncertainty on the dust-gas slopes and on $\Sigma_{d}^{\text {dif }}$ is $35 \%$ (see Appendix A).

${ }^{\text {a }} \Sigma_{I}$ is the intercept of the dust-gas relation.

${ }^{\mathrm{b}} \Sigma_{d}^{\mathrm{dif}}$ is the dust surface density corresponding to the $\mathrm{H} \mathrm{I}-\mathrm{H}_{2}$ transition.

${ }^{c} \delta^{\text {dense }}$ is the dust-gas slope in the dense ISM, where CO is detected. The slope may not constant across the surface density range of the dense phase, so the relevant, minimum values derived at the highest surface densities is quoted.

${ }^{\mathrm{d}} X_{\mathrm{CO}}^{\text {fid }}=2 \times 10^{20} \mathrm{~cm}^{-2} \mathrm{~K}^{-1} \mathrm{~km}^{-1} \mathrm{~s}$ assumed in the LMC, and $X_{\mathrm{CO}}^{\mathrm{fid}}=1 \times 10^{21} \mathrm{~cm}^{-2} \mathrm{~K}^{-1} \mathrm{~km}^{-1} \mathrm{~s}$ in the SMC. 

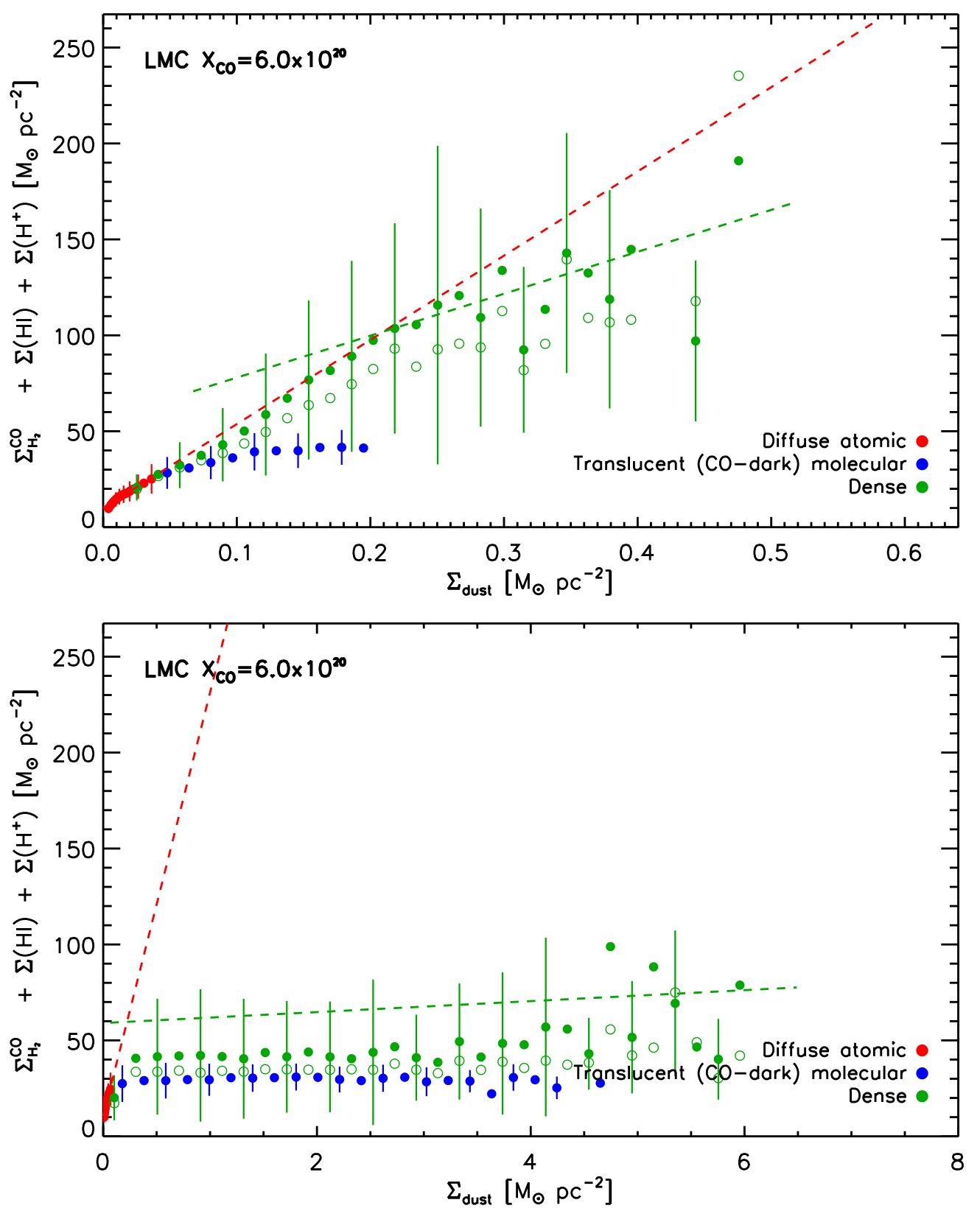

Figure 12. Pixel-to-pixel correlation (binned) between dust and total gas $\left(\mathrm{HI}, \mathrm{H}_{2}, \mathrm{H}^{+}\right)$surface densities in the LMC, with the dust surface densities derived from FIR SED fits to the SMBB model (top) and TTMBB model (bottom). The red, blue, and green points and lines correspond to the diffuse, translucent (CO-dark), and dense phases, respectively. Filled circles show the binned mean, while empty circles show the binned median. We assume our fiducial $X_{\mathrm{CO}}$ values.

(A color version of this figure is available in the online journal.)

ISM. Lower values of $X_{\mathrm{CO}}$ result in even larger variations, with $\delta^{\text {dense }}=70-130$ with a $35 \%$ systematic uncertainty for a Galactic $X_{\mathrm{CO}}$ factor.

A decrease in the dust-gas slope between the diffuse and dense ISM may be caused by coagulation of dust grains in the dense ISM, and/or accretion of gas-phase metals onto dust grains. In the first case, the GDR would stay constant and equal $\mathrm{GDR}^{\text {dif }}$, but the FIR emissivity of dust grains would increase in molecular clouds. In the second case, the dust abundance and therefore GDR would decrease from the diffuse to the dense ISM. In this case, the dense GDR would be equal to $\delta^{\text {dense }}$. Coagulation and accretion are degenerate and are expected to have similar effects on the dust-gas slope, which can be interpreted as an apparent GDR. Both processes would occur in similar density ranges, and incur apparent variations in the GDR with similar magnitudes. In a future paper, we introduce simple theoretical modeling of coagulation and accretion to constrain the magnitude of these effects, and determine how much each effect contributes to the decrease of the dust-gas slope between the diffuse and dense ISM.

This work makes use of data collected by the Herschel Space Observatory. Herschel is an ESA space observatory with science instruments provided by European-led Principal Investigator consortia and with important participation from NASA. HIPE is a joint development by the Herschel Science Ground Segment Consortium, consisting of ESA, the NASA Herschel Science Center, and the HIFI, PACS and SPIRE consortia. We acknowledge financial support from the NASA Herschel Science Center, JPL contract Nos. 1381522, 1381650, 

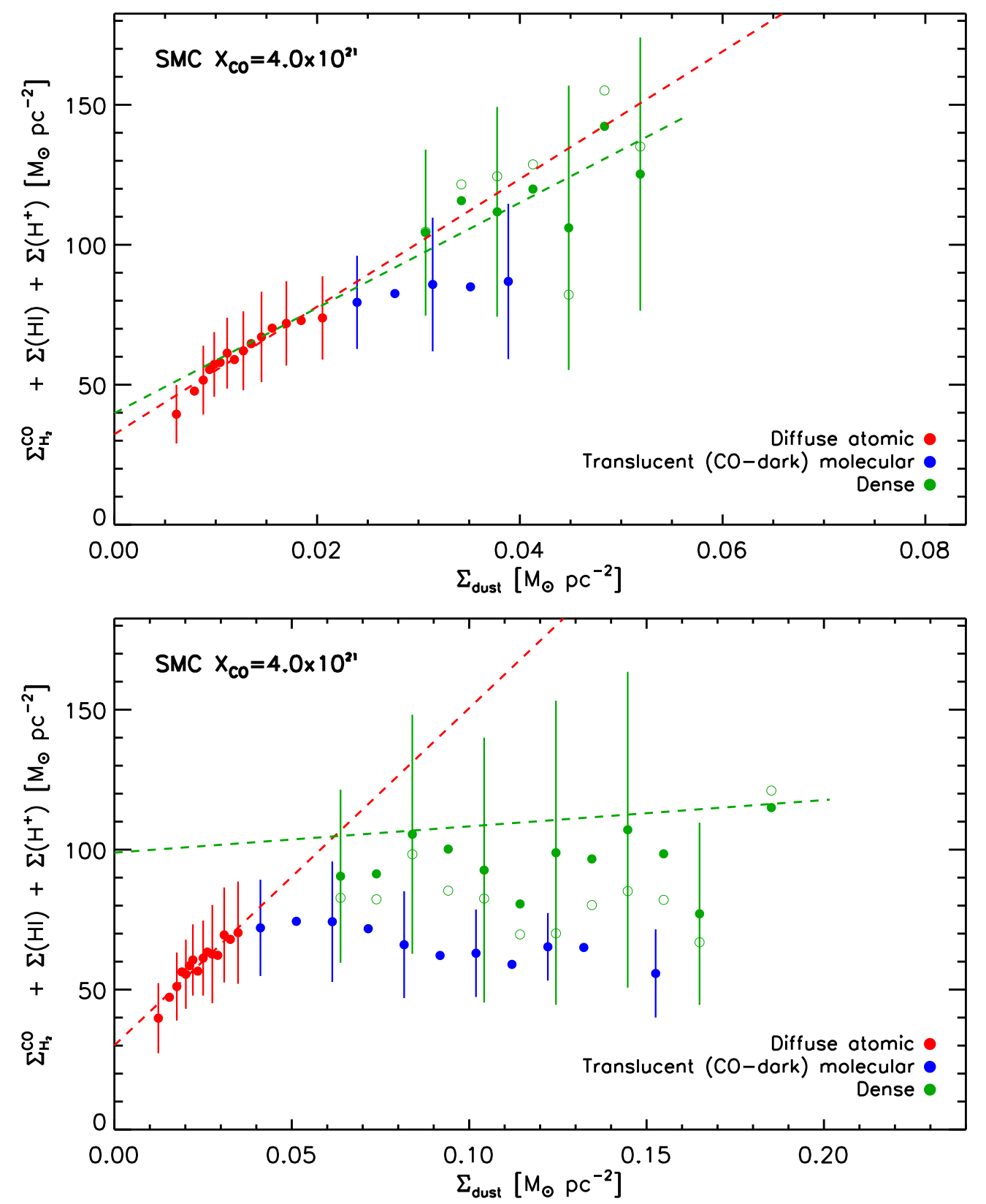

Figure 13. Pixel-to-pixel correlation (binned) between dust and total gas $\left(\mathrm{HI}, \mathrm{H}_{2}, \mathrm{H}^{+}\right)$surface densities in the SMC, with the dust surface densities derived from FIR SED fits to the SMBB model (top) and TTMBB model (bottom). The red, blue, and green points and lines correspond to the diffuse, translucent (CO-dark), and dense phases, respectively. Filled circles show the binned mean, while empty circles show the binned median. We assume our fiducial $X_{\mathrm{CO}}$ values.

(A color version of this figure is available in the online journal.)

and 1350371. SG acknowledges financial support from the Deutsche Forschungsgemeinschaft (DFG) via SFB 881 "The Milky Way System" (sub-projects B1, B2 and B8).

\section{APPENDIX A}

\section{SYSTEMATIC UNCERTAINTY ON THE DUST-GAS SLOPES}

The FIR emissivity of dust grains is not well constrained. Paper I calibrates the dust emissivity in the Herschel bands by imposing the condition that the dust surface density derived from a solar neighborhood SED fit yields a GDR of 150 that is compatible with the known depletions for this gas surface density. The random error on the calibration of $\kappa_{160}$ is $\pm 1.5 \mathrm{~cm}^{2} \mathrm{~g}^{-1}$ for the BEMBB model. This random error on $\kappa_{160}$ translates into a systematic uncertainty of $13 \%$ on the dust surface density measurement in the LMC and SMC.

The dust composition and subsequent FIR emissivity in the LMC and SMC is not necessarily identical to that in the MW. Potential differences in the dust properties between the MW and the Magellanic Clouds are an additional source of systematic uncertainty on the FIR emissivity, which Paper I estimates to be \pm 2.5 or $22 \%$ on $\kappa_{160}$.

The systematic uncertainty on the dust surface density determination, and consequently on the GDR, due to the uncertainty on the dust emissivity is thus $35 \%$. We emphasize that we are interested in the variations of the GDR with surface density and between ISM phases, not so much in its absolute value. A sensitivity analysis on the dust surface density derivation described in Figure 2 of Paper I demonstrates that there is no bias 

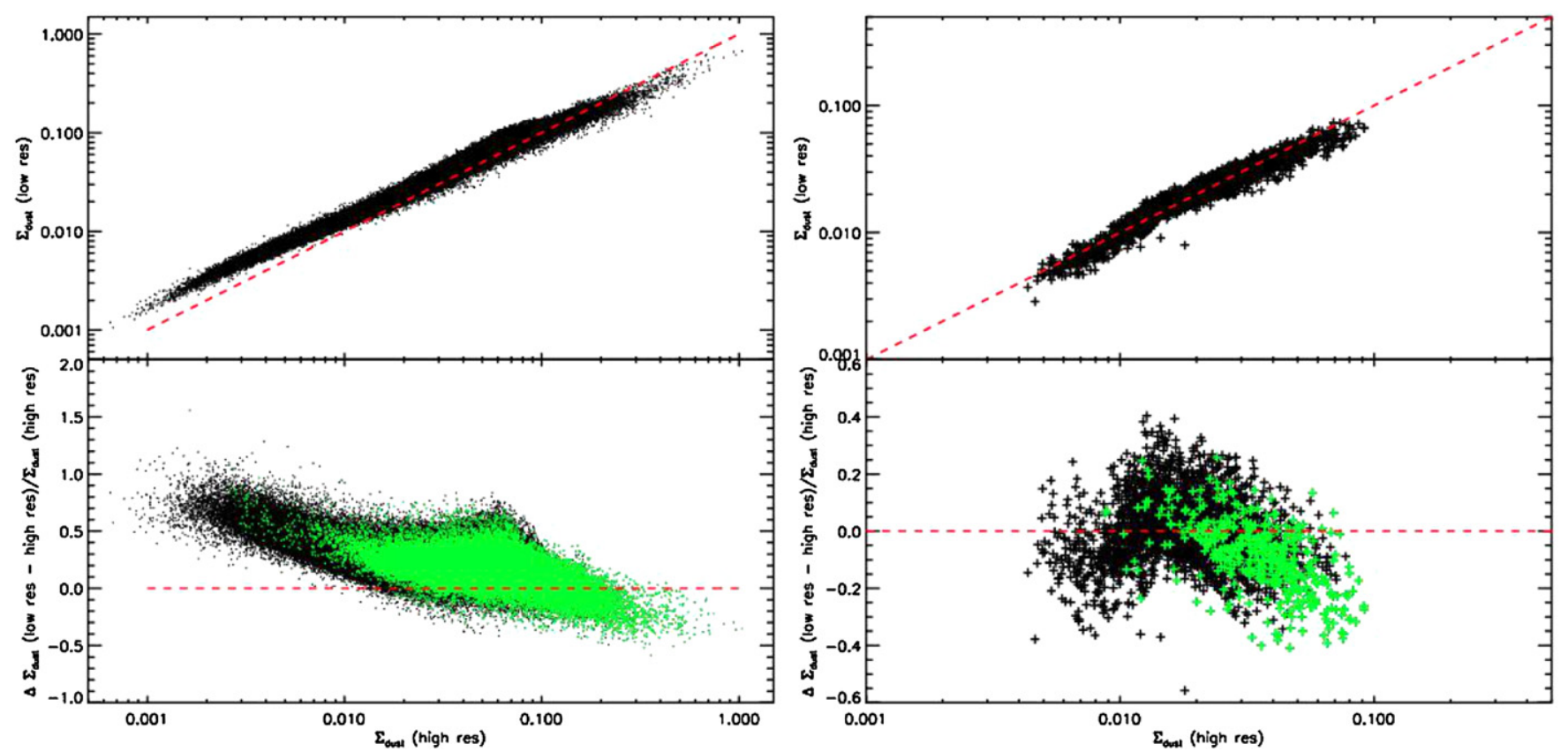

Figure 14. Comparison of the "low res" (convolution from Herschel native resolution to limiting resolution applied to the FIR images before SED fitting) and "high res" (convolution applied to the dust surface density maps after SED fitting) dust surface density maps obtained with the BEMBB model, in the LMC (left) and SMC (right). The top panels show the pixel-pixel comparison, while the bottom panels show the fractional difference between the two types of maps. The dashed lines indicate a 1:1 relation. In the bottom panels, black points correspond to pixels with no $\mathrm{CO}$ detections while $\mathrm{CO}$ is detected in the green points.

(A color version of this figure is available in the online journal.)

in the estimation of the dust surface density, and hence, that we should be able to recover the relative variations in the dust surface density and GDR accurately.

\section{APPENDIX B}

\section{EFFECT OF DIFFERENT FITTING TECHNIQUES}

We chose to use a $\chi^{2}$ minimization with respect to a linear function to derive the dust-H I and dust-total gas slopes. Other fitting methods are available, and yield significantly different results. The range of slopes obtained with different methods informs us on the systematic uncertainty associated with the fitting method. In addition to the linear fit to the pixels, we have also tried a variety of fitting methods.

For the dust-H I relation, we fit a two-dimensional Gaussian to the linear distribution of pixels in $\Sigma_{\text {dust }}-\Sigma\left(\mathrm{HI}_{\mathrm{I}}\right)$ space $\left(\mathrm{GDR}_{\text {gauss }}^{\text {dif }}\right)$, taking the orientation of the semi-major axis to be the slope of the dust-H I relation. We also fit a linear function (using a $\chi^{2}$ minimization) to the binned mean ( $\left.\mathrm{GDR}_{\text {mean }}^{\mathrm{dif}}\right)$ and median $\left(\mathrm{GDR}_{\mathrm{med}}^{\mathrm{dif}}\right)$. Last, we determined the bisector of the dust-H I distribution $\left(\mathrm{GDR}_{\mathrm{bisect}}^{\mathrm{dif}}\right)$. The resulting best fits are shown in Figure 10 and reported in Table 3. In the LMC, the resulting diffuse atomic GDR ranges from 380 (linear fit to the pixels) to 540 (bisector and two-dimensional Gaussian fits). In the SMC, we find atomic GDR values ranging from 1200 (linear fit to the pixels) to 2100 (two-dimensional Gaussian fit). These different methods are not associated with a strong bias $(\leqslant 10 \%-20 \%$, see Table 3), except for the two-dimensional Gaussian fit in the SMC (55\%).

For the dust-total gas relation in the dense phase, we performed similar fitting methods: a fit to the binned mean, the binned median, and a bisector fit to the pixel distribution. We performed the fit above the threshold dust surface density where the dust-total gas relation appears linear, as determined in Section 5. The resulting dust-gas slopes are reported in Table 4, for the fiducial $\left(X_{\mathrm{CO}}^{\mathrm{fid}}\right)$ and maximum $\left(X_{\mathrm{CO}}^{\max }\right)$ values of the $X_{\mathrm{CO}}$ factor. As an example, we show the different fits for the LMC in Figure 11.

Additionally, we performed Monte-Carlo simulations in each case to estimate the bias and random error associated with each fitting method. The resulting values, uncertainties, and biases are summarized in Tables 3 and 4 for the dust-H I and dust-total gas in the dense phase, respectively.

The width of the derived GDR and dust-gas slopes range illustrates the difficulty in characterizing the low $\mathrm{S} / \mathrm{N}$ dust-gas relation by a linear function. Not only are the distributions very scattered, but also they are probably not intrinsically linear. At low surface densities, a possible low-metallicity gas component steepens the dust-H I relation, while at high surface densities, the $\mathrm{H}-\mathrm{H}_{2}$ transition flattens it out. Different methods weight the different regions of the distribution differently, which results in a range of slopes. There is no clearly motivated choice between these different methods, and so together they provide a reasonable range for the atomic GDRs in the LMC and SMC.

\section{APPENDIX C}

\section{CONSTRAINTS ON DUST PROPERTIES}

We have investigated the GDR in the LMC and SMC using the dust surface densities derived from Herschel SED fitting to a blackbody modified by a broken emissivity law (the BEMBB model in Paper I). The dust surface density and temperature depend on the model used in the SED fitting procedure. Paper I also derived dust surface density maps for two other models: a single modified blackbody with a pure power-law spectral emissivity (SMBB), and a two-temperature component modified blackbody (TTMBB). Those different models reflect different properties of the dust grains.

We performed the same analysis as described in Section 3.1 for these additional dust models. The corresponding dust-gas 

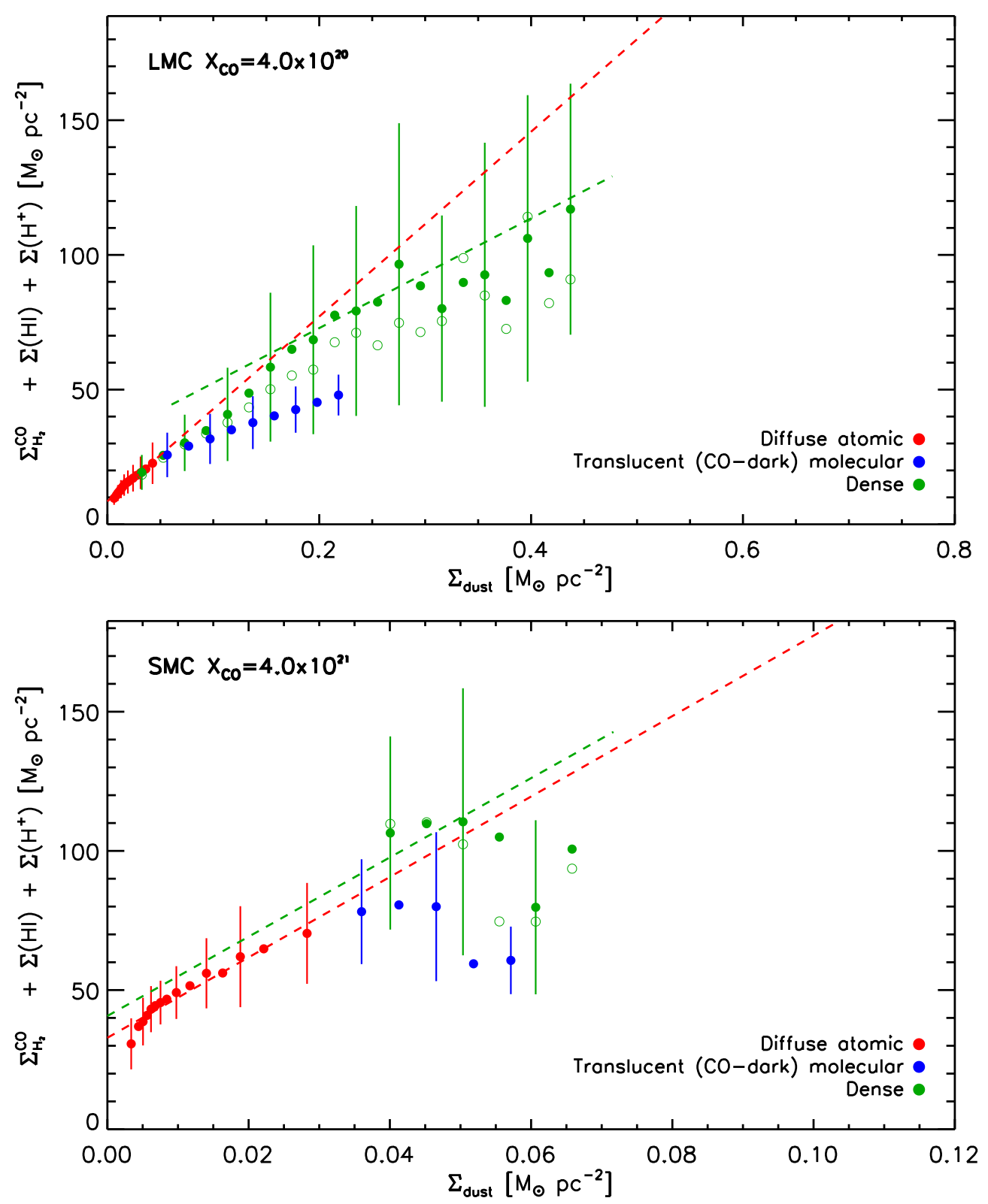

Figure 15. Pixel-to-pixel correlation (binned) between dust and total gas $\left(\mathrm{H}_{\mathrm{I}}, \mathrm{H}_{2}, \mathrm{H}^{+}\right)$surface densities in the LMC (top) and SMC (bottom), as in Figures 5 and 6 , but with the dust surface density obtained by fitting the Herschel/HERITAGE photometry after convolving the FIR maps to the limiting resolution of $1^{\prime}$ (LMC) and 2.6 (SMC). Red, blue, and green points correspond to the diffuse atomic, translucent, and dense (CO detected) phases, respectively. The filled and empty circles show the binned mean and median. We assume our $X_{\mathrm{CO}}^{\max }$ values, which are found to be $4 \times 10^{20} \mathrm{~cm}^{-2} \mathrm{~K}^{-1} \mathrm{~km}^{-1} \mathrm{~s}$ in the $\mathrm{LMC}_{\text {and }} 4 \times 10^{21} \mathrm{~cm}^{-2} \mathrm{~K}^{-1} \mathrm{~km}^{-1} \mathrm{~s}$ in the SMC when the FIR maps are convolved before performing the dust surface density derivation from SED fitting.

(A color version of this figure is available in the online journal.)

relations are shown in Figures 12 and 13. The parameters of the dust-H I relation fitting and of the dust-total gas relation are summarized in Table 5. Essentially, the dust-gas slopes obtained for the SMBB and BEMBB models are similar within the uncertainties. The TTMBB model yields dust-gas slopes that are un-physically small in the dense ISM, given the uncertainties, and no matter what value of $X_{\mathrm{CO}}$ is assumed. We can thus rule out the presence of a very cold dust component in the ISM.

\section{APPENDIX D}

\section{EFFECTS OF CONVOLVING THE FIR MAPS BEFORE THE SED FITTING}

Following the recommendations from Galliano et al. (2011), we convolved the dust surface density maps from Gordon et al.
(2014) from the native resolution of the FIR maps (36") to the limiting resolution ( $1^{\prime}$ for the LMC, and 2'.6 for the SMC) after performing the SED fitting. Another commonly used approach is to convolve the FIR maps to the limiting resolution prior to performing the SED fitting and dust surface density derivation. In the LMC, Galliano et al. (2011) demonstrated that the latter approach leads to a negative average bias (the total dust masses are underestimated), particularly for spatial resolutions worse than $50 \mathrm{pc}$. This effect is due to the dilution of cold regions into hotter regions and to the non-linear (exponential) relation between IR flux and dust temperature. Galliano et al. (2011) concluded that one should therefore perform the SED fitting and dust surface density or mass derivation at the highest possible resolution, where the uncertainty on the derived dust mass does not exceed this bias, and convolve the resulting surface density 
maps, which are a linear tracer of the ISM as opposed to FIR flux, instead of convolving the FIR flux maps prior to the SED fitting. This is particularly important when using a single temperature dust model, as in this work.

Galliano et al. (2011) predict that for resolutions $<50 \mathrm{pc}$, the bias in the total dust mass is $<5 \%$, and essentially negligible compared to the errors. Indeed, the total dust masses in the LMC and SMC with the convolution to the limiting resolution applied before the SED fitting are 5\% lower in the SMC and 16\% higher in the LMC compared to the total dust masses obtained by applying the SED fitting to the Herschel native resolution. This is consistent, within the errors, with the findings of Galliano et al. (2011, their Figure 6). Therefore, the total dust masses derived from our data should not be affected by this effect. Here, we examine the bias on the detailed pixel distribution incurred by performing the convolution first on the FIR maps before deriving the dust surface density maps. We stress that our goal is to estimate "how wrong" dust maps obtained by convolving the FIR maps before the SED fitting are, and in no way do we encourage using this method. We thus first convolve the Herschel HERITAGE maps to 1' (LMC) and 2'.6 (SMC) prior to applying the SED fitting code from Gordon et al. (2014).

Figure 14 compares the dust surface density maps derived with the convolution applied before the SED fitting ("low res") and after the SED fitting ("high res," the correct method), and their fractional difference. Fractional differences between the "low res" and "high res" dust surface density maps range between $-50 \%$ and $+100 \%$. At high surface densities, the "low res" dust surface density is always lower (by up to 50\%) than the "high res" version, because dense cold clouds are diluted with hotter surrounding regions. Since the fractional difference depends on surface density, we expect that the slope of the dust-gas relation changes between the "high res" and "low res" versions. The dust-gas relation in the LMC and SMC obtained from the "low res" dust surface densities is shown in Figure 15 for $X_{\mathrm{CO}}^{\mathrm{max}}$. With the "low res" dust maps, we find $X_{\mathrm{CO}}^{\max }=4 \times 10^{21} \mathrm{~cm}^{-2} \mathrm{~K}^{-1} \mathrm{~km}^{-1} \mathrm{~s}$ in the SMC as with the "high res" version, but a slightly lower $X_{\mathrm{CO}}^{\max }$ value of $4 \times$ $10^{20} \mathrm{~cm}^{-2} \mathrm{~K}^{-1} \mathrm{~km}^{-1} \mathrm{~s}$ in the LMC.

In the LMC with the "low res" version, we find $\mathrm{GDR}^{\mathrm{dif}}=$ $340 \pm 3$, and $\delta_{\mathrm{min}}^{\text {dense }}=290 \pm 8.4$ with $X_{\mathrm{CO}}^{\max }$. In comparison, the "high res" values are GDR dif $=380 \pm 3$, and $\delta_{\text {min }}^{\text {dense }}=190 \pm 16$. The systematic uncertainty is the same with both methods. The numbers are in agreement with Figure 14, which shows that the "low res" maps overestimate the amount of dust at low surface densities (hence the shallower dust-gas slope in the diffuse phase) and underestimate the dust surface densities in the dense phase (hence the steeper dust-gas slope in the dense phase). In the SMC, the "low res" dust-gas slopes are $\mathrm{GDR}^{\text {dif }}=1400 \pm 150$, and $\delta_{\mathrm{min}}^{\text {dense }}=1400 \pm 1200$ with $X_{\mathrm{CO}}^{\mathrm{max}}$. For comparison, the "high res" values are GDR dif $^{2}=1200 \pm$ 120 , and $\delta_{\min }^{\text {dense }}=1200 \pm 40$ with $X_{\mathrm{CO}}^{\mathrm{max}}$. Those numbers are also consistent with the trend observed in Figure 14. In the SMC, the difference between the native Herschel resolution and the limiting resolution is larger $\left(2^{\prime}\right)$ than in the LMC $\left(24^{\prime \prime}\right)$. This results in a larger scatter in the dense ISM dust-gas relation due to the mixing of phases by beam dilution. Correspondingly, the random error on $\delta_{\text {min }}^{\text {dense }}$ is much larger with the "low res" version $( \pm 1200)$ compared to the "high res" version $( \pm 40)$.

In a nutshell, while it is not correct to convolve FIR images before deriving dust surface densities by SED fitting, the conclusions presented in this paper are robust against one method or the other. The ISM phase separation and scatter in the dust-gas relation are however much cleaner if one derives the dust surface densities first from the best possible FIR resolution, and then convolves the resulting dust surface density maps to the limiting resolution of the data set.

\section{REFERENCES}

Bernard, J., Reach, W. T., Paradis, D., et al. 2008, AJ, 136, 919 Bladh, S., \& Höfner, S. 2012, A\&A, 546, A76

Bolatto, A. D., Jackson, J. M., \& Ingalls, J. G. 1999, ApJ, 513, 275

Bolatto, A. D., Leroy, A. K., Jameson, K., et al. 2011, ApJ, 741, 12

Bolatto, A. D., Leroy, A. K., Rosolowsky, E., Walter, F., \& Blitz, L. 2008, ApJ, 686,948

Bolatto, A. D., Simon, J. D., Stanimirović, S., et al. 2007, ApJ, 655, 212

Bolatto, A. D., Wolfire, M., \& Leroy, A. K. 2013, ARA\&A, 51, 207

Chlewicki, G. 1985, PhD thesis, Leiden Univ.

Coupeaud, A., Demyk, K., Meny, C., et al. 2011, A\&A, 535, A124

Dickey, J. M., Mebold, U., Stanimirovic, S., \& Staveley-Smith, L. 2000, ApJ, 536,756

Dickman, R. L., Snell, R. L., \& Schloerb, F. P. 1986, ApJ, 309, 326

Draine, B. T. 2009, in ASP Conf. Ser. 414, Cosmic Dust-Near and Far, ed. T. Henning, E. Grün, \& J. Steinacker (San francisco, CA: ASP), 453

Draine, B. T., Dale, D. A., Bendo, G., et al. 2007, ApJ, 663, 866

Draine, B. T., \& Salpeter, E. E. 1979, ApJ, 231, 438

Dwek, E. 1998, ApJ, 501, 643

Fox, A. J., Richter, P., Wakker, B. P., et al. 2013, ApJ, 772, 110

Fukui, Y., Kawamura, A., Minamidani, T., et al. 2008, ApJS, 178, 56

Fukui, Y., Torii, K., Onishi, T., et al. 2014, ApJ, 796, 59

Galliano, F., Hony, S., Bernard, J.-P., et al. 2011, A\&A, 536, A88

Gaustad, J. E., McCullough, P. R., Rosing, W., \& Van Buren, D. 2001, PASP, 113,1326

Glover, S. C. O., \& Mac Low, M.-M. 2011, MNRAS, 412, 337

Gordon, K., Clayton, G., Misselt, K., et al. 2003, ApJ, 594, 279

Gordon, K. D., Meixner, M., Meade, M. R., et al. 2011, AJ, 142, 102

Gordon, K. D., Roman-Duval, J., Bot, C., et al. 2014, ApJ, 797, 85

Gould, R. J., \& Salpeter, E. E. 1963, ApJ, 138, 393

Heyer, M., Krawczyk, C., Duval, J., \& Jackson, J. M. 2009, ApJ, 699, 1092

Hilditch, R. W., Howarth, I. D., \& Harries, T. J. 2005, MNRAS, 357, 304

Hill, J. K., \& Hollenbach, D. J. 1976, ApJ, 209, 445

Hollenbach, D. J., \& Tielens, A. G. G. M. 1999, RvMP, 71, 173

Hopkins, P. F. 2014, arXiv:1406.5509

Hughes, A., Wong, T., Ott, J., et al. 2010, MNRAS, 406, 2065

Israel, F. P. 1997, A\&A, 328, 471

Jenkins, E. B. 2009, ApJ, 700, 1299

Jones, A. P., Tielens, A. G. G. M., Hollenbach, D. J., \& McKee, C. F. 1994, ApJ, 433, 797

Jones, A. P., Tielens, A. G. G. M., Hollenbach, D. J., \& McKee, C. F. 1996, in Proc. of ESO Workshop, The Role of Dust in the Formation of Stars, ed. H. U. Käufl \& R. Siebenmorgen (Berlin: Springer), 419

Kim, S., Staveley-Smith, L., Dopita, M. A., et al. 2003, ApJS, 148, 473 Krumholz, M. R., McKee, C. F., \& Tumlinson, J. 2009a, ApJ, 693, 216 Krumholz, M. R., McKee, C. F., \& Tumlinson, J. 2009b, ApJ, 699, 850 Lee, M.-Y., Stanimirović, S., Douglas, K. A., et al. 2012, ApJ, 748, 75

Leroy, A., Bolatto, A., Stanimirovic, S., et al. 2007, ApJ, 658, 1027

Leroy, A. K., Bolatto, A., Bot, C., et al. 2009, ApJ, 702, 352

Leroy, A. K., Bolatto, A., Gordon, K., et al. 2011, ApJ, 737, 12

Liszt, H. S., \& Pety, J. 2012, A\&A, 541, A58

Matsuura, M., Dwek, E., Meixner, M., et al. 2011, Sci, 333, 1258

Meixner, M., Gordon, K. D., Indebetouw, R., et al. 2006, AJ, 132, 2268

Meixner, M., Panuzzo, P., Roman-Duval, J., et al. 2013, AJ, 146, 62

Mizuno, N., Rubio, M., Mizuno, A., et al. 2001a, PASJ, 53, L45

Mizuno, N., Yamaguchi, R., Mizuno, A., et al. 2001b, PASJ, 53, 971

Paradis, D., Paladini, R., Noriega-Crespo, A., et al. 2011, ApJ, 735, 6

Rémy-Ruyer, A., Madden, S. C., Galliano, F., et al. 2014, A\&A, 563, A31

Richter, P., Fox, A. J., Wakker, B. P., et al. 2013, ApJ, 772, 111

Roman-Duval, J., Jackson, J. M., Heyer, M., Rathborne, J., \& Simon, R. 2010, ApJ, 723, 492

Russell, S. C., \& Dopita, M. A. 1992, ApJ, 384, 508

Schaefer, B. E. 2008, AJ, 135, 112

Schwering, P. B. W. 1989, A\&AS, 79, 105

Schwering, P. B. W., \& Israel, F. P. 1989, A\&AS, 79, 79

Shetty, R., Glover, S. C., Dullemond, C. P., \& Klessen, R. S. 2011, MNRAS, 412, 1686

Snow, T. P., \& McCall, B. J. 2006, ARA\&A, 44, 367

Sofia, U. J., Gordon, K. D., Clayton, G. C., et al. 2006, ApJ, 636, 753 
Stanimirovic, S., Staveley-Smith, L., Dickey, J. M., Sault, R. J., \& Snowden, S. L. 1999, MNRAS, 302, 417

Subramanian, S., \& Subramaniam, A. 2009, A\&A, 496, 399

Subramanian, S., \& Subramaniam, A. 2010, A\&A, 520, A24

Subramanian, S., \& Subramaniam, A. 2012, ApJ, 744, 128

Tielens, A. G. G. M., \& Hollenbach, D. 1985, ApJ, 291, 722
Tumlinson, J., Shull, J. M., Rachford, B. L., et al. 2002, ApJ, 566, 857

van der Marel, R. P., \& Cioni, M.-R. L. 2001, AJ, 122, 1807

Welty, D. E., Xue, R., \& Wong, T. 2012, ApJ, 745, 173

Wolfire, M. G., Hollenbach, D., \& McKee, C. F. 2010, ApJ, 716, 1191

Wong, T., Hughes, A., Ott, J., et al. 2011, ApJS, 197, 16

Zhukovska, S., Gail, H.-P., \& Trieloff, M. 2008, A\&A, 479, 453 